\title{
LARGE CARDINALS WITH FORCING
}

\begin{abstract}
Akihiro Kanamori
This chapter describes, following the historical development, the investigation of large cardinal hypotheses using the method of forcing. Large cardinal hypotheses, also regarded as strong axioms of infinity, have stimulated a vast mainstream of modern set theory, and William Mitchell's chapter in this volume deals with their investigation through inner models, Menachem Kojman's chapter with their involvement in the study of singular cardinals, and Paul Larson's chapter with the accentuated direction of determinacy hypotheses. With the subject seen as a larger, integrated whole, we in this chapter incorporate aspects of these others while pursuing the directions involving forcing.

The author's other chapter in this volume, "Set Theory from Cantor to Cohen" (henceforth referred to as $\mathrm{CC}$ for convenience), had presented the historical development of set theory through to the creation of the method of forcing. Also, the author's book, The Higher Infinite [2003], provided the theory of large cardinals, including determinacy, up to a first plateau of combinatorial sophistication. In this chapter we first pick up the historical threads to large cardinals in that other chapter and pursue the developments involving forcing, thereby providing a complementary overlay to the book. There is no attempt at being comprehensive; rather we describe the earlier pivotal results and take up the more persistent themes, those that have mainly driven the subject.
\end{abstract}

\section{TO THE MODERN THEORY}

\subsection{Beginnings}

In this first section, we recall the early large cardinal landmarks, as pointed out in the author's other chapter $\mathrm{CC}$, and then continue through to the first substantive elaborations of the theory in the 1960s. CC describes and develops the basics of set theory and should serve as a general reference for concepts, notations, and publications not again given here, and [Kanamori, 2003] frames a larger mathematical context. The emergence of large cardinal hypotheses dates well back to the beginnings of set theory; in our quick recapitulation, we proceed anachronistically in the modern terms of transfinite ordinals and cardinals as formulated within set theory in the 1930s work of John von Neumann.

Felix Hausdorff, the first developer of the transfinite after Cantor, carried out a substantive, far-reaching investigation of uncountable ordertypes and cardinals in

Handbook of the History of Logic: Sets and Extensions in the Twentieth Century.

Volume editor: Akihiro Kanamori. General editors: Dov M. Gabbay, Paul Thagard and John Woods.

(C) 2011 Elsevier BV. All rights reserved. 
the first decade of the 20th Century. He brought in the basic concept of cofinality (the cofinality $\operatorname{cf}(\kappa)$ of a cardinal $\kappa$ is the least cardinal $\lambda$ such that $\kappa$ is the union of $\lambda$ sets each of cardinality less than $\kappa$ ) and the distinction between singular $(\operatorname{cf}(\kappa)<\kappa)$ and regular $(\operatorname{cf}(\kappa)=\kappa)$ cardinals. And for the first time, he broached a large cardinal concept, an uncountable, regular limit cardinal, now also known as a weakly inaccessible cardinal, as a natural closure point for cardinal limit processes. In penetrating work early in the next decade, Paul Mahlo considered hierarchies of such cardinals based on fixed-point phenomena and used for the first time the concept of stationary set. For a cardinal $\kappa, C \subseteq \kappa$ is closed unbounded (in $\kappa$ ) if it is closed, i.e. if $\alpha<\kappa$ and $\bigcup(C \cap \alpha)=\alpha$ then $\alpha \in C$, and unbounded i.e. $\bigcup C=\kappa$; and $S \subseteq \kappa$ is stationary (in $\kappa$ ) if $C \cap S \neq \emptyset$ for any closed unbounded $C \subseteq \kappa$. A cardinal $\kappa$ is now called weakly Mahlo if $\{\alpha<\kappa \mid \alpha$ is regular $\}$ is stationary in $\kappa$. Such a cardinal is weakly inaccessible, a limit of such, and so forth, and thus is conceptually transcendent of such cardinals. ${ }^{1}$

Ernst Zermelo in a remarkable 1930 paper offered his final axiomatization of set theory. Modern ZFC is recognizable, in that he incorporated the axioms of Replacement and Foundation, thereby promoting a view of the set-theoretic universe as stratified in a cumulative hierarchy $V=\bigcup V_{\alpha}$ indexed by ordinals forming a "regular class", where

$$
V_{0}=\emptyset ; V_{\alpha+1}=\mathcal{P}\left(V_{\alpha}\right) ; \text { and } V_{\delta}=\bigcup_{\alpha<\delta} V_{\alpha} \text { for limit ordinals } \delta .
$$

Zermelo provided natural models of his axioms, each a $V_{\kappa}$ where $\kappa$, when uncountable, is a (strongly) inaccessible cardinal (regular and strong limit, i.e. if $\lambda<\kappa$ then $\left.2^{\lambda}<\kappa\right)$. Zermelo in particular focused attention on strong limit cardinals, and e.g. one has correspondingly that $\kappa$ is (strongly) Mahlo if $\kappa$ is inaccessible and weakly Mahlo, when $\kappa$ is also a strongly limit of inaccessible cardinals, and so forth.

The journal volume containing Zermelo's paper also contained Stanisław Ulam's seminal paper on measurable cardinals, which would become focal among large cardinals. A filter over a set $Z$ is a family of subsets of $Z$ closed under the taking of supersets and of intersections. (Usually excluded from consideration as trivial are the principal filters, those of form $\{X \subseteq Z \mid A \subseteq X\}$ for some set $A \subseteq Z$.) An ultrafilter $U$ over $Z$ is a maximal filter over $Z$, i.e. for any $X \subseteq Z$, either $X \in U$ or else $Z-X \in U$. For a cardinal $\lambda$, a filter is $\lambda$-complete if it is closed under the taking of intersections of fewer than $\lambda$ members. Finally, an uncountable cardinal $\kappa$ is measurable iff there is a $\kappa$-complete ultrafilter over $\kappa$. Ulam was motivated by measure-theoretic considerations, and he viewed his work as about $\{0,1\}$-valued measures, the measure 1 sets being the sets in the ultrafilter. Ulam established that measurable cardinals are inaccessible, and this initial airing generated a question that was to keep the spark of large cardinals alive for the next three decades: Can the least inaccessible cardinal be measurable? ${ }^{2}$

\footnotetext{
${ }^{1}$ This foregoing paragraph elaborates the beginning of CC $\S 2.4$. CC cites publications, and as mentioned earlier, it should serve as a reference for this until we get well into our second section.

${ }^{2}$ The foregoing two paragraphs elaborate parts of CC $\S 3.2$.
} 
A decade later, Tarski provided a systematic development of these concepts in terms of ideals. An ideal over a set $Z$ is a family of subsets of $Z$ closed under the taking of subsets and of unions. This is the "dual" notion to filters; if $I$ is an ideal (resp. filter) over $Z$, then $\breve{I}=\{Z-X \mid X \in I\}$ is its dual filter (resp. ideal). An ideal is $\lambda$-complete if its dual filter is. A more familiar conceptualization in mathematics, Tarski investigated the general notion of ideal on a Boolean algebra in place of the power set algebra $P(Z)$. For an ideal $I \subseteq P(Z)$, the $I$-measure zero (negligible) sets are the members of $I$, the $I$-positive measure (non-negligible) sets are the members of $P(Z)-I$, and the $I$-measure one (all but negligible) sets are the members of the dual filter. For a regular cardinal $\lambda>\omega$, a basic example is the nonstationary ideal $\mathrm{NS}_{\lambda}$, consisting of the nonstationary subsets of $\lambda$, an ideal which is $\lambda$-complete, and the corresponding dual filter, the closed unbounded filter $\mathcal{C}_{\lambda}$.

At the very least as counterpoint to large cardinals, attention must be paid to how set theory was launched on an independent course as a distinctive field of mathematics by Kurt Gödel's 1930s construction of $L$, leading to the relative consistency of the Axiom of Choice and the Generalized Continuum Hypothesis. To formulate $L$ in the modern way as an archetypal construction, we affirm notation and concepts in connection with Tarski's later, systematic definition of satisfaction-in-a-structure.

For a first-order language, a structure $N$ interpreting that language (i.e. a specification of a domain of discourse as well as interpretations of the function and predicate symbols), a formula $\varphi\left(v_{1}, v_{2}, \ldots, v_{n}\right)$ of the language with the (free) variables as displayed, and $a_{1}, a_{2}, \ldots, a_{n}$ in the domain of $N$,

$$
N \models \varphi\left[a_{1}, a_{2}, \ldots, a_{n}\right]
$$

asserts that the formula $\varphi$ is satisfied in $N$ according to Tarski's recursive definition when $v_{i}$ is interpreted as $a_{i}$. A subset $y$ of the domain of $N$ is first-order definable over $N$ iff there is a formula $\psi\left(v_{1}, v_{2}, \ldots, v_{n+1}\right)$ and $a_{1}, a_{2}, \ldots, a_{n}$ in the domain of $N$ such that

$$
y=\left\{z \in N \mid N \models \psi\left[a_{1}, a_{2}, \ldots, a_{n}, z\right]\right\} .
$$

(The first-order definability of $k$-ary relations is analogously formulated with $v_{n+1}$ replaced by $k$ variables.)

Through Tarski's recursive definition and an "arithmetization of syntax" whereby formulas are systematically coded by natural numbers, the satisfaction relation $N \models \varphi\left[a_{1}, a_{2}, \ldots, a_{n}\right]$ for sets $N$ is definable in ZF set theory. On the other hand, by Tarski's result on the "undefinability of truth", the satisfaction relation for $V$ itself is not first-order definable in $V$.

For any set $x$ let $\operatorname{def}(x)$ denote the collection of subsets of $x$ first-order definable over the structure $\langle x, \in\rangle$ with domain $x$ and the membership relation restricted to it.

Then define:

$$
L_{0}=\emptyset ; L_{\alpha+1}=\operatorname{def}\left(L_{\alpha}\right), L_{\delta}=\bigcup\left\{L_{\alpha} \mid \alpha<\delta\right\} \text { for limit ordinals } \delta ;
$$


and the constructible universe

$$
L=\bigcup_{\alpha} L_{\alpha}
$$

With the formalizability of $\operatorname{def}(x)$, one can formalize the Axiom of Constructibility $V=L$, i.e. $\forall x(x \in L)$. To have a larger context, we affirm the following for a class $X$ : For a set-theoretic formula $\varphi, \varphi^{X}$ denotes $\varphi$ with its quantifiers restricted to $X$ and this extends to set-theoretic terms $t$ (like $\bigcup x, P(x)$, and so forth) through their definitions to yield $t^{X}$. $X$ is an inner model iff $X$ is a transitive class containing all the ordinals such that $\varphi^{X}$ is a theorem of ZF for every axiom $\varphi$ of ZF. What Gödel did was to show in ZF that $L$ is an inner model which satisfies AC and GCH. He thus established a relative consistency which can be formalized as an assertion: Con(ZF) implies Con(ZFC + GCH). Gödel also showed that for any inner model $M$ of $\mathrm{ZF}$ that $L \subseteq M$, so that $L$ is the canonically minimal inner model; it would be to counterpoint this minimality with "maximality" considerations that would further stimulate the investigation of large cardinals.

In later years Gödel speculated about the possibility of deciding propositions like $\mathrm{CH}$ outright with large cardinal hypotheses based on the heuristics of reflection, and later, generalization. In a 1946 address he suggested the consideration of "stronger and stronger axioms of infinity" and reflection down from $V$ : "Any proof of a set-theoretic theorem in the next higher system above set theory (i.e. any proof involving the concept of truth ...) is replaceable by a proof from such an axiom of infinity." In a 1947 expository article on the Continuum Problem Gödel presumed that $\mathrm{CH}$ would be shown independent from $\mathrm{ZF}$ and speculated more concretely about possibilities with large cardinals. He argued that the axioms of set theory do not "form a system closed in itself" and so the "very concept of set on which they are based suggests their extension by new axioms that assert the existence of still further iterations of the operation of 'set of'." In an unpublished footnote toward a 1966 revision of the article, Gödel acknowledged "extremely strong axioms of infinity of an entirely new kind", generalizations of properties of $\omega$ "supported by strong arguments from analogy." These heuristics would surface anew in the 1960s, when the theory of large cardinals developed a self-fueling momentum of its own, stimulated by the emergence of forcing and inner models. ${ }^{3}$

From the 1930s, the direct investigation of the transfinite as extension of number was advanced by the emergence of infinite combinatorics. The investigation of partition relations and trees would be particularly relevant for large cardinals. In modern terms, for ordinals $\alpha, \beta$, and $\delta$ and $n \in \omega$ the partition property

$$
\beta \longrightarrow(\alpha)_{\delta}^{n}
$$

is the assertion that for any partition $f:[\beta]^{n} \rightarrow \delta$ of the $n$-element subsets of $\beta$ into $\delta$ cells, there is an $H \subseteq \beta$ of order type $\alpha$ homogeneous for the partition, i.e. all the $n$-element subsets of $H$ lie in the same cell. Frank Ramsey in 1930 established the infinite Ramsey Theorem: $\omega \longrightarrow(\omega)_{i}^{n}$ for every $n, i \in \omega$.

\footnotetext{
${ }^{3}$ The foregoing paragraphs on Gödel and his work draw on CC $\S 3.4$, but also elaborate firstorder definability.
} 
A tree is a partially ordered set $T$ such that the predecessors of any element are well-ordered. The $\alpha$ th level of $T$ consists of those elements whose predecessors have order-type $\alpha$, and the height of $T$ is the least $\alpha$ such that the $\alpha$ th level of $T$ is empty. Generally, a chain in a partially ordered set is a linearly ordered subset, and an antichain is a subset consisting of pairwise incompatible elements. A cofinal branch of $T$ is a chain with elements at every non-empty level of $T$. Finally, for a cardinal $\kappa$, a $\kappa$-tree is a tree of height $\kappa$ each of whose levels has cardinality less than $\kappa$, and $\kappa$ has the tree property iff every $\kappa$-tree has a cofinal branch. König's Lemma, of 1927, is the assertion that $\omega$ has the tree property.

Djuro Kurepa, in his 1935 thesis, carried out the first systematic study of transfinite trees. In modern terms, an Aronszajn tree is an $\omega_{1}$-tree without a cofinal branch, i.e. a counterexample to the tree property for $\omega_{1}$. Kurepa gave Nachman Aronszajn's proof that there is an Aronszajn tree. A Suslin tree is an $\omega_{1}$-tree with no uncountable chains or antichains. Kurepa reduced a question of Mikhail Suslin to Suslin's Hypothesis, that there are no Suslin trees. Finally, a Kurepa tree is an $\omega_{1}$-tree with at least $\omega_{2}$ cofinal branches, and Kurepa's Hypothesis, the assertion that there are such trees.

Paul Erdős was the prominent figure of a strong Hungarian tradition in combinatorics, and he and his collaborators simply viewed the transfinite as a rich source of combinatorially interesting problems. One of the early advances was the 1943 Erdös-Tarski paper, which concluded with an intriguing list of six combinatorial problems, the positive solution to any, as it was to turn out, amounting to the existence of a large cardinal. In a footnote various implications were noted, one of them being essentially that for inaccessible $\kappa$, the tree property for $\kappa \mathrm{im}$ plies $\kappa \longrightarrow(\kappa)_{2}^{2}$, generalizing Ramsey's $\omega \longrightarrow(\omega)_{2}^{2}$ and making explicit the König Lemma property needed. ${ }^{4}$

\subsection{Model-theoretic methods}

The investigation of large cardinal hypotheses was crucially and essentially informed by the infusion of model-theoretic methods into set theory. Model theory was developed most concertedly by Tarski and his students in the 1950s and 1960s at the University of California at Berkeley. They carefully laid out satisfaction-ina-structure; theories (deductively closed collections of sentences) and their models; algebratization with Skolem functions and hulls; and elementary substructures and embeddings. For structures $\mathcal{A}$ and $\mathcal{B}$ for the same language, $j: \mathcal{A} \rightarrow \mathcal{B}$ is an elementary embedding if for any $a_{1}, \ldots, a_{n}$ from the domain of $\mathcal{A},\left\langle a_{1}, \ldots, a_{n}\right\rangle$ satisfies in $\mathcal{A}$ the same formulas that $\left\langle j\left(a_{1}\right), \ldots, j\left(a_{n}\right)\right\rangle$ does in $\mathcal{B}$; and when $j$ is the identity, $\mathcal{A}$ is an elementary substructure of $\mathcal{B}$, denoted $\mathcal{A} \prec \mathcal{B}$. The construction of models freely used transfinite methods and soon led to new questions in set theory, but also set theory was to be decisively advanced by the infusion of model-theoretic methods. In particular, Gödel's pivotal work with $L$ years earlier would be recast in systematic terms and generalized.

\footnotetext{
${ }^{4}$ The foregoing four paragraphs draw on $\mathrm{CC} \S 3.5$.
} 
A precursory result was a 1949 generalization by Andrzej Mostowski of the Mirimanoff-von Neumann result that every well-ordered set is order-isomorphic to exactly one ordinal with membership. A set $X$ is transitive if $\bigcup X \subseteq X$, i.e. $X$ is "closed under membership". A binary relation $R$ on a set $X$ is extensional if distinct members of $X$ have distinct $R$-predecessors, and well-founded if every non-empty $Y \subseteq X$ has an $R$-minimal element (or, assuming AC, there is no infinite $R$-descending sequence). If $R$ is an extensional, well-founded relation on a set $X$, then there is a unique transitive set $T$ and an isomorphism of the structure $\langle X, R\rangle$ onto $\langle T, \in\rangle$, i.e. a bijection $\pi: X \rightarrow T$ such that for any $x, y \in X$, $x R y$ iff $\pi(x) \in \pi(y) .\langle T, \in\rangle$ is the transitive collapse of $X$, and $\pi$ the collapsing isomorphism.

András Hajnal and Azriel Levy, in their theses of the mid-1950s, developed generalizations of $L$ that were to become basic in a richer setting. For a set $A$, Hajnal formulated the constructible closure $L(A)$ of $A$, i.e. the smallest inner model $M$ such that $A \in M$, and Levy formulated the class $L[A]$ of sets constructible relative to $A$, i.e. the smallest inner model $M$ such that for every $x \in M, A \cap x \in M$. To formulate $L(A)$, define: $L_{0}(A)=$ the smallest transitive set $\supseteq\{A\}$ (to ensure that the resulting class is transitive); $L_{\alpha+1}(A)=\operatorname{def}\left(L_{\alpha}(A)\right) ; L_{\delta}(A)=\bigcup_{\alpha<\delta} L_{\alpha}(A)$ for limit $\delta>0$; and finally $L(A)=\bigcup_{\alpha} L_{\alpha}(A)$. To formulate $L[A]$, first let $\operatorname{def}^{A}(x)$ denote the collection of subsets of $x$ first-order definable over $\langle x, \in, A \cap x\rangle$, i.e. $A \cap x$ is now interpreting a predicate that can be used in the definitions. Then define: $L_{0}[A]=\emptyset ; L_{\alpha+1}[A]=\operatorname{def}^{A}\left(L_{\alpha}[A]\right) ; L_{\delta}[A]=\bigcup_{\alpha<\delta} L_{\alpha}[A]$ for limit $\delta>0 ;$ and finally $L[A]=\bigcup_{\alpha} L_{\alpha}[A]$. With the "trace" $\bar{A}=A \cap L[A]$ one has $L_{\alpha}[\bar{A}]=L_{\alpha}[A]$ for every $\alpha$ and so $L[\bar{A}]=L[A]$.

$L(A)$ realizes the algebraic idea of building up a model starting from a set of generators, and $L[A]$ the logical idea of building up a model using $A$ construed as a predicate. $L(A)$ may not satisfy $\mathrm{AC}$ since it may not have a well-ordering of $A$, yet $L[A]$ always satisfies that axiom. This distinction was only to surface later, as both Hajnal and Levy took $A$ to be a set of ordinals, when $L(A)=L[A]$, and used these models to establish conditional independence results of the sort: if the failure of $\mathrm{CH}$ is consistent, then so is that failure together with $2^{\lambda}=\lambda^{+}$for sufficiently large cardinals $\lambda$. In the coming expansion of the 1960s, both Hajnal and Levy would be otherwise engaged, with Hajnal becoming a major combinatorial set theorist and collaborator with Erdős, and Levy, a pioneer in the investigation of independence results.

The set-theoretic generalization of first-order logic allowing transfinitely indexed logical operations clarified the size of measurable cardinals. Extending familiarity by abstracting to a new domain, Tarski in 1962 formulated the strongly compact and weakly compact cardinals by ascribing natural generalizations of the key compactness property of first-order logic to the corresponding infinitary languages. These cardinals had figured in the 1943 Erdős-Tarski paper in equivalent combinatorial formulations that were later seen to imply that a strongly compact cardinal is measurable, and a measurable cardinal is weakly compact. Tarski's student Hanf then established, using the satisfaction relation for infinitary languages, that there 
are many inaccessible cardinals (and Mahlo cardinals) below a weakly compact cardinal. A fortiori, the least inaccessible cardinal is not measurable. This breakthrough was the first result about the size of measurable cardinals since Ulam's original 1930 paper and was greeted as a spectacular success for metamathematical methods. Hanf's work radically altered size intuitions about problems coming to be understood in terms of large cardinals and ushered in model-theoretic methods into the study of large cardinals beyond the Mahlo cardinals. Weak compactness was soon seen to have a variety of characterizations, most notably, $\kappa$ is weakly compact iff $\kappa \rightarrow(\kappa)_{2}^{2}$ iff $\kappa \rightarrow(\kappa)_{\lambda}^{n}$ for every $n \in \omega$ and $\lambda<\kappa$ iff $\kappa$ is inaccessible and has the tree property, and this was an early, significant articulation of the large cardinal extension of context for effecting known proof ideas and methods. ${ }^{5}$

The concurrent emergence of the ultraproduct construction in model theory set the stage for the development of the modern theory of large cardinals. The construction was brought to the forefront by Tarski and his students after Jerzy Eoś's 1955 adumbration of its fundamental theorem, and the new method of constructing concrete models brought set theory and model theory even closer together in a surge of results and a lasting interest in ultrafilters.

The ultraproduct construction was driven by the algebraic idea of making a structure out of a direct product of structures as modulated (or "reduced") by a filter. The particular case when all the structures are the same, the ultrapower, was itself seen to be substantive. To briefly describe a focal case for set theory, let $N$ be a set, construed as a structure with $\in$, and $U$ an ultrafilter over a set $Z$. On ${ }^{Z} N$, the set of functions from $Z$ to $N$, define

$$
f={ }_{U} g \text { iff }\{i \in Z \mid f(i)=g(i)\} \in U .
$$

The filter properties of $U$ imply that $=_{U}$ is an equivalence relation on ${ }^{Z} N$, so with $(f)_{U}$ denoting the corresponding equivalence class of $f$, set ${ }^{Z} N / U=\left\{(f)_{U} \mid f \in\right.$ $\left.{ }^{Z} N\right\}$. Next, the filter properties of $U$ show that a binary relation $E_{U}$ on ${ }^{Z} N / U$ can be unambiguously defined by

$$
(f)_{U} E_{U}(g)_{U} \quad \text { iff } \quad\{i \in Z \mid f(i) \in g(i)\} \in U .
$$

$={ }_{U}$ is thus a congruence relation, one that preserves the underlying structure; this sort of preservation is crucial in ultraproduct and classical, antecedent constructions with filters. The ultrapower of $N$ by $U$ is then defined to be the structure $\left\langle{ }^{Z} N / U, E_{U}\right\rangle$. The crux of the construction is the fundamental Eos's Theorem: For a formula $\varphi\left(v_{1}, \ldots, v_{n}\right)$ and $f_{1}, \ldots, f_{n} \in{ }^{Z} N$,

$$
\begin{array}{r}
\left\langle{ }^{Z} N / U, E_{U}\right\rangle \models \varphi\left[\left(f_{1}\right)_{U}, \ldots,\left(f_{n}\right)_{U}\right] \quad \text { iff } \\
\qquad\left\{i \in Z \mid \mathcal{N} \models \varphi\left[f_{1}(i), \ldots, f_{n}(i)\right]\right\} \in U .
\end{array}
$$

Satisfaction in the ultrapower is thus reduced to satisfaction on a large set of coordinates, large in the sense of $U$. The proof is by induction on the complexity

\footnotetext{
${ }^{5}$ The foregoing paragraphs of this subsection draw on CC $\S 3.6$.
} 
of $\varphi$ using the filter properties of $U$, the ultrafilter property for the negation step, and $\mathrm{AC}$ for the existential quantifier step.

$E_{U}$ is an extensional relation, and crucially, well-founded when $U$ is $\aleph_{1}$-complete. In that case by Mostowski's theorem there is a collapsing isomorphism $\pi$ of the ultrapower onto the transitive collapse $\langle M, \in\rangle$. Moreover, if for $x \in N, c_{x}$ is the constant function: $N \rightarrow\{x\}$ and $j_{U}: N \rightarrow M$ is defined by $j_{U}(x)=\pi\left(\left(c_{x}\right)_{U}\right)$, then $j_{U}$ is an elementary embedding, i.e. for any formula $\varphi\left(v_{1}, \ldots, v_{n}\right)$ and $a_{1}, \ldots, a_{n} \in$ $N$,

$$
\langle N, \in\rangle \models \varphi\left[a_{1}, \ldots, a_{n}\right] \quad \text { iff }\langle M, \in\rangle \models \varphi\left[j_{U}\left(a_{1}\right), \ldots, j_{U}\left(a_{n}\right)\right]
$$

by Łoś's Theorem. When we have well-foundedness, the ultrapower is identified with its transitive collapse and denoted $\operatorname{Ult}(N, U)$.

All of the foregoing is applicable, and will be applied, with proper classes $N$, as long as we replace the equivalence class $(f)_{U}$ by sets

$$
(f)_{U}^{0}=\left\{g \in(f)_{U} \mid g \text { has minimal rank }\right\}
$$

("Scott's trick"), and take Łoś's Theorem as a schema for formulas.

The model theorist H. Jerome Keisler established penetrating connections between combinatorial properties of ultrafilters and of their ultraproducts, and in particular took the ultrapower of a measurable cardinal $\kappa$ by a $\kappa$-complete ultrafilter over $\kappa$ to provide a new proof of Hanf's result that there are many large cardinals below a measurable cardinal. With Ulam's concept shown in a new light as providing well-founded ultrapowers, Dana Scott then struck on the idea of taking the ultrapower of the entire universe $V$ by a $\kappa$-complete ultrafilter over a measurable $\kappa$, exploiting the resulting well-foundedness to get an elementary embedding $j: V \rightarrow \operatorname{Ult}(V, U)$. Importantly, $\kappa$ is the critical point, i.e. $j(\alpha)=\alpha$ for every $\alpha<\kappa$ yet $\kappa<j(\kappa)$ : Taking e.g. the identity function id $: \kappa \rightarrow \kappa$, $\{\xi<\kappa \mid \alpha<\xi<\kappa\} \in U$ for every $\alpha<\kappa$, so that $\kappa \leq \pi\left((\mathrm{id})_{U}\right)<j(\kappa)$ by Loś's Theorem. If $V=L$, then $\operatorname{Ult}(V, U)=L$ by the definability properties of $L$, but this confronts $\kappa<j(\kappa)$, e.g. if $\kappa$ were the least measurable cardinal. (One could also appeal to the general fact that $U \notin \operatorname{Ult}(V, U)$; that one "loses" the ultrafilter when taking the ultrapower would become an important theme in later work.) With this Scott established that if there is a measurable cardinal, then $V \neq L$. Large cardinal assumptions thus assumed a new significance as a means for "maximizing" possibilities away from Gödel's canonical, delimitative construction.

The ultrapower construction provided one direction of a new characterization that established a central structural role for measurable cardinals: There is an elementary embedding $j: V \rightarrow M$ for some $M$ with critical point $\delta$ iff $\delta$ is a measurable cardinal. Keisler provided the converse direction: With $j$ as hypothesized, $U_{j} \subseteq P(\delta)$ defined "canonically" by $X \in U_{j}$ iff $\delta \in j(X)$ is a $\delta$-complete ultrafilter over $\delta$. Generating ultrafilters thus via "ideal" elements would become integral to the theory of ultrafilters and large cardinals.

This characterization, when viewed with the focus on elementary embeddings, raises a point that will be even more germane, and thus will be emphasized later, 
in connection with strong hypotheses. That a $j: V \rightarrow M$ is elementary is not formalizable in set theory because of the appeal to the satisfaction relation for $V$, let alone the assertion that there is such a class $j$. Thus the "characterization" is really one of giving a formalization, one that provides operative sense through the ultrapower construction. Ulam's original concept was thus made intrinsic to set theory with the categorical imperative of elementary embeddings. In any event ZFC is never actually transcended in consistency results; one can always work in a sufficiently large $V_{\alpha}$.

In Scott's $j: V \rightarrow M=\operatorname{Ult}(V, U)$ the concreteness of the ultrapower construction delivered ${ }^{\kappa} M \subseteq M$, i.e. $M$ is closed under the taking of arbitrary (in $V$ ) $\kappa$-sequences, so that in particular $V_{\kappa+1} \cap M=V_{\kappa+1}$. Through this agreement strong reflection conclusions can be drawn. $U$ is normal iff $\pi\left((\mathrm{id})_{U}\right)=\kappa$, i.e. the identity function is a "least non-constant" function, a property that can be easily arranged. For such $U$, since $\kappa$ is inaccessible, it is so in $M$ and hence by Łoś's Theorem $\{\xi<\kappa \mid \xi$ is inaccessible $\} \in U$ - the inaccessible cardinals below $\kappa$ have measure one. An analogous argument applies to any $V_{\kappa+1}$ property of $\kappa$ like weak compactness, and so, as would typify large cardinal hypotheses, measurability articulates its own sense of reflective transcendence over "smaller" large cardinals.

Normality went on to become staple to the investigation of ideals and large cardinals. Formulated for an ideal $I$ over a cardinal $\lambda, I$ is normal iff whenever a function $f$ is regressive on an $S \in P(\lambda)-I$, there is a $T \in P(S)-I$ on which $f$ is constant. The 1956 Fodor's Lemma is the assertion that the nonstationary ideal $\mathrm{NS}_{\lambda}$ is normal for regular $\lambda>\omega$, and a multitude of "smallness" properties other than nonstationarity has been seen to lead to normal ideals.

Through model-theoretic methods set theory was brought to the point of entertaining elementary embeddings into well-founded models. It was soon to be transfigured by a new means for getting well-founded extensions of well-founded models. ${ }^{6}$

\section{THE ADVENT OF FORCING}

\subsection{Cohen}

Paul Cohen (1934-2007) in April 1963 established the independence of AC from ZF and the independence of $\mathrm{CH}$ from ZFC. That is, Cohen established that Con(ZF) implies Con $(\mathrm{ZF}+\neg \mathrm{AC})$ and that Con(ZFC) implies Con(ZFC $+\neg \mathrm{CH})$. Already prominent as an analyst, Cohen had ventured into set theory with fresh eyes and an open-mindedness about possibilities. These results solved two central problems of set theory. But beyond that, Cohen's proofs were the inaugural examples of a new technique, forcing, which was to become a remarkably general and flexible method for extending models of set theory. Forcing has strong intuitive underpinnings and reinforces the notion of set as given by the first-order ZF axioms with prominent

\footnotetext{
${ }^{6}$ The foregoing paragraphs on the ultraproduct construction considerably elaborates the latter part of CC $\S 3.6$, by setting out the basics of the construction.
} 
uses of Replacement and Foundation. If Gödel's construction of $L$ had launched set theory as a distinctive field of mathematics, then Cohen's method of forcing began its transformation into a modern, sophisticated one.

Cohen's approach was to start with a model $M$ of ZF and adjoin a set $G$ that witnesses some desired new property. This would have to be done in a minimal fashion in order that the resulting extension $M[G]$ also model ZF, and so Cohen devised special conditions on both $M$ and $G$. Cohen's key idea was to tie $G$ closely to $M$ through a partially ordered system of sets in $M$ called conditions that would approximate $G$. While $G$ may not be a member of $M, G$ is to be a subset of some $Y \in M$ (with $Y=\omega$ a basic case), and these conditions would "force" assertions about the eventual $M[G]$, e.g. by deciding some of the membership questions, whether $x \in G$ or not, for $x \in Y$. The assertions are to be just those expressible in a ramified language with terms developed in $M$, and Cohen developed a corresponding forcing relation $p \Vdash \varphi$, " $p$ forces $\varphi$ ", between conditions $p$ and formulas $\varphi$, a relation with properties reflecting his approximation idea. For example, if $p \Vdash \varphi$ and $p \Vdash \psi$, then $p \Vdash \varphi \wedge \psi$. The conditions are ordered according to the constraints they impose on the eventual $G$, so that if $p \Vdash \varphi$, and $q$ is a stronger condition, then $q \Vdash \varphi$. It was crucial to Cohen's approach that the forcing relation, like the ramified language, be definable in $M$.

The final ingredient which gives this whole scaffolding life is the incorporation of a certain kind of set $G$. Stepping out of $M$ and actually assuming that it is countable, Cohen enumerated the formulas of the ramified language in a countable sequence and required that $G$ be completely determined by a sequence of stronger and stronger conditions $p_{0}, p_{1}, p_{2}, \ldots$ such that for every formula $\varphi$ of the ramified language exactly one of $\varphi$ or $\neg \varphi$ is forced by some $p_{n}$. Such a $G$ is called a generic set. Cohen was able to show that the resulting $M[G]$ does indeed satisfy the axioms of ZF: Every assertion about $M[G]$ is already forced by some condition; the forcing relation is definable in $M$; and so the $\mathrm{ZF}$ axioms holding in $M$, most crucially Replacement and Foundation, can be applied to the ramified terms and language to derive corresponding forcing assertions about the $\mathrm{ZF}$ axioms holding in $M[G]$.

Cohen first described the case when $G \subseteq \omega$ and the conditions $p$ are functions from some finite subset of $\omega$ into $\{0,1\}$ and $p \Vdash \dot{n} \in \dot{G}$ if $p(n)=1$ and $p \Vdash \dot{n} \notin \dot{G}$ if $p(n)=0$. Today, a $G$ so adjoined to $M$ is called a Cohen real over $M$. If subsets of $\omega$ are identified with reals as traditionally construed, that $G$ is generic can be extrinsically characterized by saying that $G$ meets every open dense set of reals lying in $M$. Generally, a $G \subseteq \kappa$ analogously adjoined with conditions of cardinality less than $\kappa$ is called a Cohen subset of $\kappa$. Cohen established the independence of $\mathrm{CH}$ by adjoining a set which in effect is a sequence of many Cohen reals. It was crucial that the cardinals in the ground model and generic extension coincide, and Cohen to this end drew out and relied on the important countable chain condition (c.c.c.): Any antichain, i.e. collection of mutually incompatible conditions, is countable (where two forcing conditions said to be incompatible if they have no common, stronger condition). 
Several features of Cohen's arguments would quickly be reformulated, reorganized, and generalized, but the thrust of his approach through definability and genericity would remain. Cohen's great achievement lies in devising a concrete procedure for extending well-founded models of set theory in a minimal fashion to well-founded models of set theory with new properties but without altering the ordinals.

Just in the first weeks after Cohen's discovery, Solomon Feferman, who had been extensively consulted by Cohen as he was coming up with forcing, established further independences elaborating $\neg \mathrm{AC}$ and about definability. Levy soon joined in this work and pursued both directions, formulating the "Levy collapse" of an inaccessible cardinal, and Stanley Tennenbaum established the failure of Suslin's Hypothesis by generically adjoining a Suslin tree with finite conditions.

The Levy collapse would become of central importance for large cardinals. Cohen had shown how to "collapse" a cardinal: Given a regular cardinal $\kappa$ and $\alpha>\kappa$ in a ground model $M$, a generic surjection $G$ of $\alpha$ onto $\kappa$ can be adjoined using as conditions approximations of cardinality less than $\kappa$. In the Levy collapse of an inaccessible cardinal $\lambda>\kappa$, such collapsing functions are collectively adjoined for every $\alpha<\lambda$, with the inaccessibility of $\lambda$ ensuring that it becomes the successor cardinal to $\kappa$ in the generic extension. The Levy collapse of an inaccessible cardinal to make it $\omega_{1}$ or $\omega_{2}$, the first or second uncountable cardinal of the extension, would become basic to subsequent developments.

The extent and breadth of the expansion of set theory henceforth described dwarfs all that has been described before, both in terms of the numbers of people involved and the results established. With clear intimations of a new and concrete way of building models, set theorists rushed in and, with forcing becoming method, were soon establishing a cornucopia of relative consistency results, truths in a wider sense, with some illuminating classical problems of mathematics. ZFC became quite unlike Euclidean geometry and much like group theory, with a wide range of models being investigated for their own sake. ${ }^{7}$

\subsection{Solovay and forcing}

Robert Solovay above all epitomized this period of sudden expansion in set theory with his mathematical sophistication and central results about and with forcing, and in the areas of large cardinals and descriptive set theory. Following initial graduate study in differential topology, Solovay turned to set theory after hearing a May 1963 lecture by Cohen. Just weeks after, Solovay elaborated the independence of $\mathrm{CH}$ by characterizing the possibilities for the size of $2^{\kappa}$ for regular $\kappa$ and made the first exploration of a range of cardinals. Building on this William Easton in late 1963 established the definitive result for powers of regular cardinals: Suppose that $\mathrm{GCH}$ holds and $F$ is a class function from the class of regular cardinals to cardinals such that for regular $\kappa \leq \lambda, F(\kappa) \leq F(\lambda)$ and the cofinality $\mathrm{cf}(F(\kappa))>\kappa$. Then there is a (class) forcing extension preserving cofinalities in which $2^{\kappa}=F(\kappa)$

\footnotetext{
${ }^{7}$ The foregoing subsection draws on CC $\S 4.1$, but also elaborates on the Levy collapse.
} 
for every regular $\kappa$. Thus, as Solovay had seen locally, the only restriction beyond monotonicity on the power function for regular cardinals is that given by a wellknown constraint, the classical Zermelo-König inequality that $\operatorname{cf}\left(2^{\kappa}\right)>\kappa$ for any cardinal $\kappa$. Easton's result enriched the theory of forcing with the introduction of proper classes of forcing conditions, the basic idea of a product analysis, and the now familiar concept of Easton support. The result focused interest on the possibilities for powers of singular cardinals and the Singular Cardinals Hypothesis (SCH), which asserts that $2^{\kappa}$ for singular $\kappa$ is the least possible with respect to the powers $2^{\mu}$ for $\mu<\kappa$ as given by monotonicity and the Zermelo-Konnig inequality. This requires in particular that for singular strong limit cardinals $\kappa, 2^{\kappa}=\kappa^{+}$. Easton's models satisfy SCH, and the Singular Cardinals Problem, to determine the range of possibilities for powers of singular cardinals, would become a major stimulus for the further development of set theory much as the Continuum Problem had been for its early development.

In the Spring of 1964 Solovay established a result remarkable for its mathematical depth and revelatory of what standard of argument was possible with forcing: If there is an inaccessible cardinal, then in a $\mathrm{ZF}$ inner model of a forcing extension the Principle of Dependent Choices (DC) holds and every set of reals is Lebesgue measurable, has the Baire property, and has the perfect set property. Solovay's inner model is precluded from having a well-ordering of the reals, but DC is a choice principle implying the regularity of $\omega_{1}$ and sufficient for the formalization of the traditional theory of measure and category on the real numbers. Thus, Solovay's work vindicated the early descriptive set theorists in the sense that the regularity properties can consistently hold for all sets of reals in a bona fide model for the classical mathematical analysis of the reals. To prove his result Solovay applied the Levy collapse of an inaccessible cardinal to make it $\omega_{1}$. For the Lebesgue measurability he introduced a new kind of forcing beyond Cohen's direct ways of adjoining new sets of ordinals or collapsing cardinals, that of adding a random real, given by forcing with the Borel sets of positive measure as conditions and $p$ stronger than $q$ when $p-q$ is null. In contrast to Cohen reals, a random real meets every measure one subset of the unit interval lying in the ground model. Solovay's work not only opened the door to a wealth of different forcing arguments, but to this day his original definability arguments remain vital to descriptive set theory.

The perfect set property, central to Cantor's direct approach to the Continuum Problem through definability, led to the first acknowledged instance of a new phenomenon in set theory: the derivation of equi-consistency results between large cardinal hypotheses and combinatorial propositions about low levels of the cumulative hierarchy. Forcing showed just how relative the Cantorian concept of cardinality is, since bijective functions could be adjoined to models of set theory and powers like $2^{\aleph_{0}}$ can be made arbitrarily large with relatively little disturbance. For instance, large cardinals were found to satisfy substantial propositions even after they are Levy collapsed to $\omega_{1}$ or $\omega_{2}$. Conversely, such propositions were found to entail large cardinal propositions in an $L$-like inner model, mostly pointedly the very same initial large cardinal hypothesis. Thus, for some large cardinal property 
$\varphi(\kappa)$ and proposition $\psi$, there is a direction $\operatorname{Con}(\exists \kappa \varphi(\kappa)) \rightarrow \operatorname{Con}(\psi)$ established by a collapsing forcing argument, and a converse direction $\operatorname{Con}(\psi) \rightarrow \operatorname{Con}(\exists \kappa \varphi(\kappa))$ established by witnessing $\varphi(\kappa)$ in an inner model.

Solovay's result provided the forcing direction from an inaccessible cardinal to the proposition that every set of reals has the perfect set property and $\omega_{1}$ is regular. But Ernst Specker in 1957 had in effect established that if this obtains, then $\omega_{1}$ (of $V$ ) is inaccessible in $L$. Thus, Solovay's use of an inaccessible cardinal was actually necessary, and its collapse to $\omega_{1}$ complemented Specker's observation. The emergence of such equi-consistency results is a subtle realization of earlier hopes of Gödel for deciding propositions via large cardinals. Forcing, however, quickly led to the conclusion that there could be no direct implication for $\mathrm{CH}$ itself: Levy and Solovay, also in 1964, established that measurable cardinals neither imply nor refute $\mathrm{CH}$, with an argument generalizable to other inaccessible large cardinals. Rather, $\mathrm{CH}$ and many other propositions would be reckoned with in terms of consistency, the methods of forcing and inner models being the operative modes of argument.

Building on his Lebesgue measurability result Solovay in 1965 reactivated the classical descriptive set theory program of investigating the extent of the regularity properties (in the presence of AC) by providing characterizations in terms of forcing and definability concepts for the $\boldsymbol{\Sigma}_{2}^{1}$ sets, the level at which Gödel through his work on $L$ had in fact established from $V=L$ the failure of the properties. This led to the consistency relative to ZFC of the Lebesgue measurability of all $\boldsymbol{\Sigma}_{2}^{1}$ sets. Also, the characterizations showed that the regularity properties for $\boldsymbol{\Sigma}_{2}^{1}$ sets follow from existence of a measurable cardinal. Thus, although measurable cardinals do not decide $\mathrm{CH}$, they do establish the perfect set property for $\boldsymbol{\Sigma}_{2}^{1}$ sets so that "CH holds for the $\boldsymbol{\Sigma}_{2}^{1}$ sets". 89

In a separate initiative, Solovay $[1966 ; 1971]$ established the equi-consistency of the existence of a measurable cardinal and the "real-valued" measurability of $2^{\aleph_{0}}$, i.e. that there is a (countably additive) measure extending Lebesgue measure to all sets of reals. For the forcing direction, Solovay starting with a measurable cardinal adjoined random reals and applied the Radon-Nikodym Theorem of analysis, and for the converse direction, he starting with a real-valued measure enlisted the inner model constructed relative to the ideal of measure zero sets. This consistency result provided context for an extended investigation of the possibilities for the continuum as structured by such a measure. Through this work the concept of saturated ideal, first studied by Tarski, was brought to prominence as a generalization of having a measurable cardinal applicable to the low levels of the cumulative hierarchy. For an ideal over a cardinal $\kappa, I$ is $\lambda$-saturated iff for any $\left\{X_{\alpha} \mid \alpha<\lambda\right\} \subseteq P(\kappa)-I$ there are $\beta<\gamma<\lambda$ such that $X_{\beta} \cap X_{\gamma} \in P(\kappa)-I$ (i.e. the

\footnotetext{
${ }^{8}$ A coda after many years: Although Solovay's use of an inaccessible cardinal for universal Lebesgue measurability seemed $a d$ hoc at the time, in 1979 Saharon Shelah [1984] established in a tour de force that if $\mathrm{ZF}+\mathrm{DC}$ and all $\Sigma_{3}^{1}$ sets of reals are Lebesgue measurable, then $\omega_{1}$ is inaccessible in $L$.

${ }^{9}$ The foregoing paragraphs of $\S 2.2$ draws on CC $\S 4.1$ and $\S 4.2$. We now proceed anew, citing publications, for the rest of this chapter.
} 
corresponding Boolean algebra has no antichains of cardinality $\lambda$ ). The ideal of measure zero sets is $\aleph_{1}$-saturated, and Solovay showed that if $I$ is any $\kappa$-complete $\lambda$-saturated ideal over $\kappa$ for some $\lambda<\kappa$, then $L[I] \models$ " $\kappa$ is measurable".

Solovay's work also brought to the foreground the concept of generic ultrapower and generic elementary embedding. For an ideal $I$ over $\kappa$, forcing with the members of $P(\kappa)-I$ as conditions and $p$ stronger than $q$ when $p-q \in I$ engenders an ultrafilter on the ground model $P(\kappa)$. With this one can construct an ultrapower of the ground model in the generic extension and a corresponding elementary embedding. It turns out that the $\kappa^{+}$-saturation of the ideal ensures that this generic ultrapower is well-founded. Thus, a synthesis of forcing and ultrapowers is effected, and this raised enticing possibilities for having such large cardinal-type structure low in the cumulative hierarchy.

The development of the theory of forcing went hand in hand with this procession of central results. Solovay had first generalized forcing to arbitrary partial orders of conditions, proceeding in terms of incompatible members and dense sets and Levy's concept of generic filter. In his work on the Baire property for his 1964 model, Solovay came to the idea of assigning values to formulas from a complete Boolean algebra. Loosely speaking, the value would be the supremum of all the conditions forcing it. Working independently, Solovay and Scott developed the idea of recasting forcing entirely in terms of Boolean-valued models. This approach showed how to replace Cohen's ramified languages by a more direct induction on rank and how to avoid his dependence on a countable model. Boolean-valued functions play the role of sets, and formulas involving these functions are assigned Boolean-values by recursion respecting logical connectives and quantifiers. By establishing in ZFC that e.g. there is a complete Boolean algebra assigning the formula expressing $\neg \mathrm{CH}$ Boolean value one, a semantic construction was replaced by a syntactic one that directly secured relative consistency.

Still, the view of forcing as a way of actually extending models held the reservoir of sense and the promise of discovery, and after Shoenfield [1971] popularized an approach to the forcing relation that captured the gist of the Boolean-valued approach, forcing has been generally cast as a matter of partial orders and generic filters. Boolean algebras would nonetheless underscore and enhance the setting: partial orders are to have a maximum element 1; one is attuned to the separativity of partial orders, the property that unique Boolean completions; Boolean-values are used when illuminating; and embedding results for forcing partial orders are cast, as most algebraically informative, in terms of Boolean algebras.

By the 1970s there would be a further assimilation of both the syntactic and semantic approaches, in that generic extensions would be "taken" of $V$. In this the current approach, a partial order $\langle P,<\rangle$ of conditions is specified to a purpose, with $p<q$ for $p$ being stronger than $q$. A class $V^{P}$ of $P$-names defined recursively is used in forcing assertions, with a canonical name $\check{x}$ corresponding to $x \in V$. A $D \subseteq P$ is dense if for any $p \in P$ there is a $d \in D$ with $d \leq p$. An $F \subseteq P$ is a filter if (i) if $p \in F$ and $p \leq q$, then $q \in F$, and (ii) if $p_{1}, p_{2} \in F$ then there is an $r \in F$ with $r \leq p_{1}$ and $r \leq p_{2}$. Finally, $G \subseteq P$ is a $V$-generic filter if $G$ is a 
filter such that for every dense $D \subseteq P, G \cap D \neq \emptyset$. One posits such a $G$ and takes a generic extension $V[G]$, its properties argued for on the basis of combinatorial properties of $P$. For inner or transitive set models $M$, one proceeds analogously to define $M$-generic filters meeting every dense set belonging to $M$ and takes generic extensions $M[G]$.

In this one goes against the sense of $V$ as the universe of all sets and Tarski's "undefinability of truth", but actually $V$ has become schematic for a ground model. Generic extensions of inner models $M$ are taken with $M$-generic $G$, and moreover, successive iterated extensions are taken, exacerbating any preoccupation with a single universe of sets. As the techniques of forcing were advanced, the methodology was itself soon to be woven into set theory as part of its postulations.

Solovay and Tennenbaum earlier in 1965 (cf. their [1971]) had established the consistency of Suslin's Hypothesis, that there are no Suslin trees, illuminating a classical question from 1920; they had made ground-breaking use of iterated forcing to "kill" Suslin trees by forcing with them as partial orders and shooting cofinal branches through them. D. Anthony Martin pointed out that the SolovayTennenbaum argument actually established the consistency of a closure of forcing extensions of a certain kind, an instrumental "axiom" now known as Martin's Axiom (MA): For any countable-chain-condition partial order $P$ and collection $\mathcal{D}$ of fewer than $2^{\aleph_{0}}$ dense subsets of $P$, there is a filter $G \subseteq P$ meeting every member of $\mathcal{D}$. Thus method became axiom, and many consistency results could now be simply stated as direct consequences of a single umbrella proposition. $\mathrm{CH}$ technically implies MA, but the Solovay-Tennenbaum argument established the consistency of MA with the continuum being arbitrarily large.

While classical results with $\mathrm{CH}$ had worked on an $\aleph_{0} / \aleph_{1}$ dichotomy, MA established a $<2^{\aleph_{0}} / 2^{\aleph_{0}}$ dichotomy. For example, [Martin and Solovay, 1970] established that MA implies that the union of fewer than $2^{\aleph_{0}}$ Lebesgue measure zero sets is again Lebesgue measure zero. Sierpiński in 1925 had established that every $\boldsymbol{\Sigma}_{2}^{1}$ set of reals is the union of $\aleph_{1}$ Borel sets. Hence, MA and $2^{\aleph_{0}}>\aleph_{1}$ implies that every $\boldsymbol{\Sigma}_{2}^{1}$ set of reals is Lebesgue measurable. Many further results plied the $<2^{\aleph_{0}} / 2^{\aleph_{0}}$ dichotomy to show that under MA inductive arguments can be carried out in $2^{\aleph_{0}}$ steps that previously succeeded under $\mathrm{CH}$ in $\aleph_{1}$ steps. The Continuum Problem was newly illuminated as a matter of method, by showing that $\mathrm{CH}$ as a construction principle could be generalized to $2^{\aleph_{0}}$ being arbitrarily large.

Glancing across the wider landscape, forcing provided new and diverse ways of adjoin generic reals and other sets, and these led to new elucidations, for example about cardinal characteristics, or invariants, of the continuum and combinatorial structures and objects, like ultrafilters over $\omega$. The work on Suslin's Hypothesis in hand and with the possibilities afforded by Martin's Axiom, the investigation of general topological notions gathered steam. With Mary Ellen Rudin and her students at Wisconsin breaking the ground, new questions were raised for general topological spaces about separation properties, compactness-type covering properties, separability and metrizability, and corresponding cardinal characteristics. 


\section{$2.30^{\#}$ and $L[U]$}

The infusion of forcing into set theory induced a broad context extending beyond its applications and sustained by model-theoretic methods, a context which included central developments about large cardinals having their source in Scott's 1961 result that measurable cardinals contradict $V=L$. Haim Gaifman [1964] invented iterated ultrapowers and established seminal results about and with the technique, results which most immediately stimulated definitive work in the formative theses of Silver and Kunen.

Jack Silver in his 1966 Berkeley thesis (cf. his [1971c]) provided a structured sense of transcendence over $L$ in terms of the existence of a special set of natural numbers $0^{\#}$ ("zero sharp") which refined an earlier formulation of Gaifman and was quickly investigated by Solovay [1967] in terms of definability. Mostowski and Andrzej Ehrenfeucht in their [1956] had developed theories whose models have indiscernibles, implicitly ordered members of the domain all of whose $n$-tuples satisfy the same formulas. They had applied Ramsey's Theorem in compactness arguments to get models generated by indiscernibles, models consequently having many automorphisms. Silver applied partition properties satisfied by measurable cardinals to produce indiscernibles within given structures, particularly in the initial segment $\left\langle L_{\omega_{1}}, \in\right\rangle$ of the constructible universe. With definability and Skolem hull arguments, Silver was able to isolate a canonical collection of sentences to be satisfied by indiscernibles, a theory whose models cohere to get $L$ itself as generated by canonical ordinal indiscernibles - a dramatic accentuation of the original Gödel generation of $L .0^{\#}$ is that theory coded as a real, and as Solovay emphasized, $0^{\#}$ is the only possible real to satisfy a certain $\Pi_{2}^{1}$ relation, one whose complexity arises from its asserting that to every countable well-ordering there corresponds a well-founded model of the coded theory. The canonical class, closed and unbounded, of ordinal indiscernibles is often called the Silver indiscernibles. Having these indiscernibles substantiates $V \neq L$ in drastic ways: Each indiscernible $\iota$ has various large cardinal properties and satisfies $L_{\iota} \prec L$, so that by a straightforward argument the satisfaction relation for $L$ is definable from $0^{\#}$. The theory of $0^{\#}$ was seen to relativize, and for reals $a \in{ }^{\omega} \omega$ the analogous $a^{\#}$ for the inner model $L[a]$ would play focal roles in descriptive set theory as based on definability.

Kunen's main large cardinal results emanating from his 1968 Stanford thesis (cf. [1970]) would be the definitive structure results for inner models of measurability. For $U$ a normal $\kappa$-complete ultrafilter over a measurable cardinal $\kappa$, the inner model $L[U]$ of sets constructible relative to $U$ is easily seen with $\bar{U}=U \cap L[U]$ to satisfy $L[U]=$ " $\bar{U}$ is a normal $\kappa$-complete ultrafilter". With no presumption that $\kappa$ is measurable (in $V$ ) and taking $U \in L[U]$ from the beginning, call $\langle L[U], \in, U\rangle$ a $\kappa$-model iff $\langle L[U], \in, U\rangle \models$ " $U$ is a normal $\kappa$-complete ultrafilter over $\kappa$ ". Solovay observed that in a $\kappa$-model, the GCH holds above $\kappa$ by a version of Gödel's argument for $L$ and that $\kappa$ is the only measurable cardinal by a version of Scott's argument. Silver [Silver, 1971a] then established that the full GCH holds, thereby establishing the relative consistency of GCH and measurability; Silver's 
proof turned on a local structure $L_{\alpha}[U]$ being acceptable in the later parlance of inner model theory.

Kunen made Gaifman's technique of iterated ultrapowers integral to the subject of inner models of measurability. For a $\kappa$-model $\langle L[U], \in, U\rangle$, the ultrapower of $L[U]$ by $U$ with corresponding elementary embedding $j$ provides a $j(\kappa)$-model $\langle L[j(U)], \in, j(U)\rangle$, and this process can be repeated. At limit stages, one can take the direct limit of models, which when well-founded can be identified with the transitive collapse. Indeed, by Gaifman's work these iterated ultrapowers are always well-founded, i.e. $\kappa$-models are iterable. Kunen showed that the $\lambda$ th iterate of a $\kappa$-model for any regular $\lambda>\kappa^{+}$is of form $\left\langle L\left[\mathcal{C}_{\lambda}\right], \in, \mathcal{C}_{\lambda} \cap L\left[\mathcal{C}_{\lambda}\right]\right\rangle$, where $\mathcal{C}_{\lambda}$ again is the closed unbounded filter over $\lambda$, so that remarkably, constructing relative to a filter definable in set theory leads to an inner model of measurability. With this, there can be comparison of $\kappa$-models and $\kappa^{\prime}$-models by iterating them up to a sufficiently large $\lambda$. This comparison possibility led to the structure results: (1) for any $\kappa$-model and $\kappa^{\prime}$-model with $\kappa<\kappa^{\prime}$, the latter is an iterated ultrapower of the former, and (2) for any $\kappa$, there is at most one $\kappa$-model. It then followed that if $\kappa$ is measurable and $U_{1}$ and $U_{2}$ are any $\kappa$-complete ultrafilters over $\kappa$, then $L\left[U_{1}\right]=L\left[U_{2}\right]$. These various results argued forcefully for the coherence and consistency of the concept of measurability. As for the methods employed, in the subsequent development of inner model theory iterability and comparison would remain as basic features of the subject.

Kunen importantly emphasized that iterated ultrapowers can be taken of an inner model $M$ with respect to an ultrafilter $U$ even if $U \notin M$, as $\operatorname{long} U$ is an $M$ ultrafilter, i.e. $U$ in addition to having $M$ related ultrafilter properties also satisfies an "amenability" condition for $M$. A crucial dividend was a characterization of the existence of $0^{\#}$ that secured its central importance in inner model theory. With $0^{\#}$, any increasing shift of the Silver indiscernibles provides an elementary embedding $j: L \rightarrow L$. Kunen established conversely that such an embedding generates indiscernibles, so that $0^{\#}$ exists iff there is a (non-identity) elementary embedding $j: L \rightarrow L$. Starting with such an embedding Kunen defined the corresponding ultrafilter $U$ over the critical point and showed that $U$ is an $L$-ultrafilter with which the iterated ultrapowers of $L$ are well-founded. The successive images of the critical point were seen to be indiscernibles for $L$, giving $0^{\#}$. As inner model theory was to develop, this sharp analysis would become schematic: the "sharp" of an inner model $M$ would encapsulate transcendence over $M$, and the non-rigidity of $M$, that there is a (non-identity) elementary embedding $j: M \rightarrow M$, would provide equivalent structural sense.

\subsection{Jensen and constructibility}

These various results were set against a backdrop of an increasing articulation of Gödel's original notion of constructibility. Levy [1965] had put forward the appropriate hierarchy for the first-order formulas of set theory: A formula is $\Sigma_{0}$ and $\Pi_{0}$ if it is bounded, i.e. having only quantifiers expressible in terms of $\forall v \in w$ 
and $\exists v \in w$, and recursively, a formula is $\Sigma_{n+1}$ if it is of the form $\exists v_{1} \ldots \exists v_{k} \varphi$ where $\varphi$ is $\Pi_{n}$ and is $\Pi_{n+1}$ if it is of the form $\forall v_{1} \ldots \forall v_{k} \varphi$ where $\varphi$ is $\Sigma_{n}$. Two basic points about discounting bounded quantifiers are that $\Sigma_{0}$ formulas are absolute for transitive structures, i.e. they hold in such structures just in case they hold in $V$, and that if $\varphi$ is $\Sigma_{n}$ (resp. $\Pi_{n}$ ) then $\exists v \in w \varphi$ and $\forall v \in x \varphi$ are equivalent in ZFC to $\Sigma_{n}\left(\right.$ resp. $\left.\Pi_{n}\right)$ formulas by uses of Replacement.

Gödel's original GCH result with $L$ was newly seen in light of the structured context for definability. For $N$ and $M$ construed as structures with $\in, j: N \rightarrow M$ is a $\Sigma_{n}$-elementary embedding iff for any $\Sigma_{n} \varphi\left(v_{1}, \ldots, v_{k}\right)$ and $x_{1}, \ldots, x_{k} \in N$, $N \models \varphi\left[x_{1}, \ldots, x_{k}\right]$ iff $M \models \varphi\left[j\left(x_{1}\right), \ldots, j\left(x_{k}\right)\right] . N$ is a $\Sigma_{n}$-elementary substructure of $M$, denoted $N \prec_{n} M$, iff the identity map is $\Sigma_{n}$-elementary. Analysis of the satisfaction relation established that being an $L_{\alpha}$ is a $\Sigma_{1}$ property, and this led to the Condensation Lemma:

If $\alpha$ is a limit ordinal and $N \prec_{1} L_{\alpha}$, then the transitive collapse of $N$ is $L_{\beta}$ for some $\beta \leq \alpha$.

Operatively, one applies this lemma with Skolem's algebraic approach to logic by taking $N$ to be a $\Sigma_{1}$-Skolem hull in $L_{\alpha}$ : For any $\Sigma_{0}$ formula $\varphi\left(v_{1}, \ldots, v_{n}, v_{n+1}\right)$ and $x_{1}, \ldots, x_{n} \in L_{\alpha}$, if $\left\langle L_{\alpha}, \in\right\rangle \models \varphi\left[x_{1}, \ldots x_{n}, y\right]$ for $y \in L_{\alpha}$, let $f_{\varphi}\left(x_{1}, \ldots, x_{n}\right)$ be such a $y$. Then let $N$ be the algebraic closure of some subset of $L_{\alpha}$ under these Skolem functions. The road from the Condensation Lemma to Gödel's Fundamental Theorem for the consistency of GCH is short. Generally, the lemma articulates a crucial hierarchical cohesion, and its various emanations would become fundamental to all inner model theory.

The consummate master of constructibility was to be Ronald Jensen, whose first systematic analysis transformed the subject with the introduction of the fine structure theory for $L$. Jensen's work is distinguished by the persistent pursuit of internal logical structure, the sophistication of the local apparatus developed, and a series of remarkable successes with reverberations throughout the whole expanse of set theory. Jensen [1968] made an initial breakthrough by showing that $V=L$ implies the failure of Suslin's Hypothesis, i.e. (there is a Suslin tree) ${ }^{L}$, applying $L$ for the first time after Gödel to establish a relative consistency result about a classical proposition. The initial breach had been when Tennenbaum in 1963 (cf. his [1968]) had adjoined a Suslin tree with forcing with finite conditions, and then Thomas Jech [1967] had provided another forcing argument using countable conditions; Jensen at first pitched his construction in the guise of a forcing argument, one in fact like Tennenbaum's. This is the paradigmatic case of what would become a recurring phenomenon: A combinatorial existence assertion is first shown to be relatively consistent with ZFC using forcing, and then that assertion is shown to hold in $L$, the minimal inner model.

The lack of cofinal branches in Suslin trees is complemented by their abundance in Kurepa trees. An early, eye-catching forcing result was due to Silver [1971b]: If there is an inaccessible cardinal, then in the extension by the Levy collapse to make it $\omega_{2}$, Kurepa's Hypothesis fails, i.e. there are no Kurepa trees. Notably, 
in a 1966 masters' thesis D. Stewart [1966] showed how to adjoin a Kurepa tree by forcing. Inspired by Jensen's Suslin tree construction, the ubiquitous Solovay established: (there is a Kurepa tree) ${ }^{L}$. Moreover, it was noted that if $\omega_{2}$ (of $V$ ) is not inaccessible in $L$, then Solovay's argument still works to establish that there is a Kurepa tree. Thus, another equi-consistency result illuminated a classical issue: The failure of Kurepa's Hypothesis is equi-consistent with having an inaccessible cardinal.

Jensen, and others, isolated the combinatorial features of $L$ that enabled these Suslin and Kurepa constructions, and in 1969 he together with Kunen worked out a larger theory. The focus was mainly on two combinatorial principles for a regular cardinal $\kappa, \diamond_{\kappa}$ ("diamond") and a strengthening, $\diamond_{\kappa}^{+}$("diamond plus"). Stating the first,

$\nabla_{\kappa} \quad$ There is a sequence $\left\langle S_{\alpha} \mid \alpha<\kappa\right\rangle$ with $S_{\alpha} \subseteq \alpha$ such that for any $X \subseteq \kappa,\left\{\alpha<\kappa \mid X \cap \alpha=S_{\alpha}\right\}$ is stationary in $\kappa$.

Just $\diamond$ is implicitly $\nabla_{\omega_{1}}$. $\nabla_{\kappa}$ implies $\bigcup_{\alpha<\kappa} \kappa^{|\alpha|}=\kappa$ (so that $\diamond$ implies CH) as every bounded subset of $\kappa$ occurs in a $\nabla_{\kappa}$ sequence. Indeed, a $\nabla_{\kappa}$ sequence is an enumeration of the bounded subsets of $\kappa$ that can accommodate every $X \subseteq \kappa$ in anticipatory constructions where $X \cap \alpha$ appearing in the enumeration for many $\alpha$ 's suffices. Within a few years $\diamond$ would be on par with $\mathrm{CH}$ as a construction principle with wide applications in topology, algebra, and analysis. ${ }^{10}$

Jensen abstracted his Suslin tree result to: (1) if $V=L$, then $\nabla_{\kappa}$ holds for every regular $\kappa>\omega$, and (2) if $\nabla_{\omega_{1}}$ holds, then there is a Suslin tree. Solovay's result was abstracted to higher, $\kappa$-Kurepa trees, $\kappa$-trees with at least $\kappa^{+}$cofinal branches, in terms of a new cardinal concept, ineffability, arrived at independently by Jensen and Kunen: If $V=L$ and $\kappa>\omega$ is regular, then $\nabla_{\kappa}^{+}$holds iff $\kappa$ is not ineffable. Ineffable cardinals, stronger than weakly compact cardinals, would soon be seen to have a range of involvements and an elegant theory. As for higher Suslin trees, how they might be constructed in $L$ initially motivated a new combinatorial principle, one that soon figured in a sophisticated forcing argument.

The crowning achievement of the investigation of Suslin's Hypothesis was its joint consistency with $\mathrm{CH}$, $\mathrm{Con}(\mathrm{ZFC}) \rightarrow \mathrm{Con}(\mathrm{ZFC}+\mathrm{CH}+\mathrm{SH})$, established by Jensen (cf. [Devlin and Johnsbraten, 1974]). In the Solovay-Tennenbaum consistency proof for SH, cofinal branches had been adjoined iteratively to Suslin trees as they arose and direct limits were taken at limit stages, a limiting process that conspired to adjoin new reals so that $\mathrm{CH}$ fails. Jensen, with considerable virtuosity for the time, devised a way to kill Suslin trees less directly and effected the iteration according to a curtailed tree-like indexing - so that no new reals are ever adjoined. That indexing is captured by the $\kappa=\omega_{1}$ case of the combinatorial principle $\square_{\kappa}$ ("square"):

$\square_{\kappa} \quad$ There is a sequence $\left\langle C_{\alpha}\right| \alpha$ a limit ordinal $\left\langle\kappa^{+}\right\rangle$such that for $\alpha<\kappa^{+}$:

\footnotetext{
${ }^{10}$ Another coda of Shelah's after many years: In 2007 he established that for successors $\lambda^{+}>$ $\omega_{1}, 2^{\lambda}=\lambda^{+}$actually implies $\diamond_{\lambda^{+}}$, so that the two are equivalent.
} 
(a) $C_{\alpha} \subseteq \alpha$ is closed unbounded in $\alpha$,

(b) for $\beta$ a limit point of $C_{\alpha}, C_{\alpha} \cap \beta=C_{\beta}$, and

(c) for $\omega \leq \operatorname{cf}(\alpha)<\kappa$, the order-type of $C_{\alpha}$ is less than $\kappa$.

is immediate, as witnessed by any ladder system, i.e. a sequence $\left\langle C_{\alpha}\right| \alpha$ a limit ordinal $\left\langle\omega_{1}\right\rangle$ such that $C_{\alpha}$ is of order-type $\omega$ and cofinal in $\alpha . \square_{\kappa}$ for $\kappa>\omega$ brings out the tension between the desired (b) and the needed (c). As such, $\square_{\kappa}$ came to guide many a construction of length $\kappa^{+}$based on components of cardinality $<\kappa$.

Jensen showed that $\square_{\kappa}$ can be adjoined by straightforward forcing with initial approximations. Continuing his work on constructibility, Jensen soon established: If $V=L$, then $\square_{\kappa}$ holds for every $\kappa$. As for higher Suslin trees, a $\kappa$-Suslin tree is expectedly a $\kappa$-tree with no chains or antichains of cardinality $\kappa$. Jensen's work on these trees having actually motivated his formulation of $\square_{\kappa}$, he established, generalizing his result for $\kappa=\omega_{1}$ : (1) for any $\kappa, \diamond_{\kappa^{+}}$and $\square_{\kappa}$ imply that there is a $\kappa^{+}$-Suslin tree, and, for limit cardinals $\kappa$, the characterization (2) there is a $\kappa$-Suslin tree iff $\kappa$ is not weakly compact. It is a notable happenstance that Suslin's early, 1920 speculation would have such extended ramifications in modern set theory.

Jensen's results that $\square_{\kappa}$ holds in $L$ and (2) above were the initial applications of his fine structure theory. Unlike Gödel who had focused with $L$ on relative consistency, Jensen regarded the investigation of how the constructible hierarchy grows by examining its behavior at arbitrary levels as of basic and intrinsic interest. And with his fine structure theory Jensen developed a considerable and intricate machinery for this investigation. A pivotal question became: when does an ordinal $\alpha$ first get "singularized", i.e. what is the least $\beta$ such that there is in $L_{\beta+1}$ an unbounded subset of $\alpha$ of smaller order-type, and what $\Sigma_{n}$ definitional complexity does this set have? One is struck by the contrast between Jensen's attention to such local questions as this one, at the heart of his proof of $\square_{\kappa}$, and how his analysis could lead to major large-scale results of manifest significance.

Jensen's CH + SH consistency result established the consistency of CH together with the failure of $\nabla_{\omega_{1}}$. What about the possible failure of $\square_{\omega_{1}}$ ? Solovay observed: If a Mahlo cardinal is Levy collapsed to make it $\omega_{2}$, then in the extension $\square_{\omega_{1}}$ fails. Moreover, Jensen's argument for $\square_{\kappa}$ works to show that if $\kappa^{+}$is not Mahlo in $L$, then $\square_{\kappa}$ holds. Thus, among the increasing number of results complementing the historical emergence of large cardinal hypotheses was yet another equi-consistency result: The failure of $\square_{\omega_{1}}$ is equi-consistent to having a Mahlo cardinal.

\section{STRONG HYPOTHESES}

\subsection{Large large cardinals}

With elementary embedding having emerged as a systemic concept in set theory, Solovay and William Reinhardt at Berkeley in the late 1960s formulated inter- 
related large cardinal hypotheses stronger than measurability. Reinhardt conceived extendibility, and he and Solovay independently, supercompactness. (See the later [Solovay et al., 1978] for an exposition.) A cardinal $\kappa$ is $\gamma$-supercompact iff there is an elementary embedding $j: V \rightarrow M$ for some inner model $M$, with critical point $\kappa$ and $\gamma<j(\kappa)$ such that ${ }^{\gamma} M \subseteq M$, i.e. $M$ is closed under the taking of arbitrary $\gamma$-sequences. $\kappa$ is supercompact iff $\kappa$ is $\gamma$-supercompact for every $\gamma$. Evidently, the heuristics of generalization and reflection were at work here, as $\kappa$ is measurable iff $\kappa$ is $\kappa$-supercompact, and stronger closure properties imposed on the target model $M$ ensure stronger reflection properties. For example, if $\kappa$ is $2^{\kappa}$-supercompact with witnessing $j: V \rightarrow M$, then $M \models$ " $\kappa$ is measurable", since ${ }^{2^{\kappa}} M \subseteq M$ implies that every ultrafilter over $\kappa$ is in $M$, and so if $U_{j} \subseteq P(\kappa)$ is defined canonically from $j$ by $X \in U_{j}$ iff $\kappa \in j(X)$, then $\{\xi<\kappa \mid \xi$ is measurable $\} \in U_{j}$ by Łoś's Theorem. Supercompactness was initially viewed as an ostensible strengthening of Tarski's strong compactness in that, with the focus on elementary embedding, reflection properties were directly incorporated. Whether strong compactness is actually equivalent to supercompactness became a new, "identity crisis" issue.

Reinhardt entertained a prima facie extension of these ideas, that there is a (non-identity) elementary embedding $j: V \rightarrow V$. With suspicions soon raised, Kunen [1971a] dramatically established that this is inconsistent with ZFC by applying an Erdős-Hajnal partition relation result, a combinatorial contingency making prominent use of the Axiom of Choice. This contingency pointed out a specific lack of closure of the target model: For any elementary embedding $j: V \rightarrow M$ with critical point $\kappa$, let $\lambda$ be the supremum of $\kappa<j(\kappa)<j^{2}(\kappa)<\ldots$ Then, $V_{\lambda+1} \nsubseteq M$. This lack of closure has essentially stood as the weakest known to this day.

A net of hypotheses consistency-wise stronger than supercompactness was soon cast across the conceptual space delimited by Kunen's inconsistency. For $n \in \omega, \kappa$ is $n$-huge iff there is an elementary embedding $j: V \rightarrow M$, for some inner model $M$, with critical point $\kappa$ such that ${ }^{n}(\kappa) M \subseteq M$. As had been first formulated by Kunen, $\kappa$ is huge iff $\kappa$ is 1-huge. If $\kappa$ is huge, then $V_{\kappa}=$ "there are many supercompact cardinals". Thematically close to Kunen's inconsistency were several hypotheses articulated for further investigation, e.g. there is a (non-identity) elementary embedding $j: V_{\lambda} \rightarrow V_{\lambda}$ for some $\lambda$.

The appearance of proper classes in these various formulations raises issues about legitimacy. By Tarski's "undefinability of truth", the satisfaction relation for $V$ is not definable in $V$, and the elementary embedding characterization of measurability already suffers from this shortcoming. However, the $\gamma$-supercompactness of $\kappa$ can be analogously formulated in terms of the existence of a "normal" ultrafilter over the set mathcal $P_{\kappa} \gamma=[\gamma]^{<\kappa}=\{x \subseteq \gamma|| x \mid<\kappa\}$. Similarly, $n$-hugeness can also be recast. As for Kunen's inconsistency, his argument can be regarded as establishing: There is no (non-identity) elementary embedding $j: V_{\lambda+2} \rightarrow V_{\lambda+2}$ for any $\lambda$.

The details on $\gamma$-supercompactness drew out new, generalizing concepts for 
filters (and so, for ideals). Suppose that $Z$ is a set and $F$ a filter over $P(Z)$ (so $F \subseteq P(P(Z)))$. Then $F$ is fine iff for any $a \in Z,\{x \in P(Z) \mid a \in x\} \in F$. F is normal iff whenever $f$ is a function satisfying $\{x \in P(Z) \mid f(x) \in x\} \in F$, i.e. $f$ is a choice function on a set in $F$, there is an $a \in Z$ such that $\{x \in P(Z)$ $f(x)=a\} \in F$, i.e. $f$ is constant on a set in $F$. When $Z$ is a cardinal $\kappa$ and $\kappa=\{x \in P(\kappa) \mid x \in \kappa\} \in F$, then this new normality reduces to the previous concept. With an analogous reduction to filters over $\mathcal{P}_{\kappa} \gamma=[\gamma]^{<\kappa}$, we have the formulation: $\kappa$ is $\gamma$-supercompact iff there is a $\kappa$-complete, fine, normal ultrafilter over $\mathcal{P}_{\kappa} \gamma$. Also, Tarski's concept of a cardinal $\kappa$ being strongly compact can be formulated as being $\gamma$-compact for every $\gamma \geq \kappa$, where $\kappa$ is $\gamma$-compact iff there is a $\kappa$-complete, fine ultrafilter over $\mathcal{P}_{\kappa} \gamma$. These formulations inspired a substantial combinatorial investigation of filters over sets $\mathcal{P}_{\kappa} \gamma$, and a general, structural approach to filters over sets $P(Z)$.

Whether it is in these large cardinal hypotheses or the transition from $V$ to $V[G]$ in forcing, the appeal to the satisfaction relation for $V$ is liberal and unabashed in modern set-theoretic practice. Yet ZFC remains parsimoniously the official theory and this carries with it the necessary burden of formalization. On the other hand, it is the formalization that henceforth carries the operative sense; for example, the ultrafilter characterization of $\gamma$-supercompactness delivers through the concreteness of the ultrapower construction critical properties that become part of the concept in its use. It has become commonplace in modern set theory that informal assertions and schematic procedures often convey an incipient intentional sense, but formalization refines that sense with workable structural articulations.

\subsection{Determinacy}

Before proceeding with the historical development of the ZFC theory of large cardinals, we draw back to tuck in an account of a development from a different quarter, a development that at first seemed novel and orthogonal but eventually became woven into the larger fabric of the investigation of large cardinals as strong propositions. The investigation of the determinacy of infinite games is the most distinctive and intriguing development of modern set theory, and the correlations eventually achieved with large cardinals the most remarkable and synthetic. ${ }^{11}$ For a set $X$ and $A \subseteq{ }^{\omega} X$, let $G_{X}(A)$ denote the following "infinite two-person game with perfect information": There are two players, $I$ and $I I$. $I$ initially chooses an $x(0) \in X$; then $I I$ chooses an $x(1) \in X$; then $I$ chooses an $x(2) \in X$; then $I I$ chooses an $x(3) \in X$; and so forth:

$$
\begin{array}{llllll}
I: & x(0) & & x(2) & & \ldots \\
I I: & & x(1) & & x(3) & \ldots
\end{array}
$$

Each player before making each of his moves is privy to the sequence of previous moves ("perfect information"); and the players together specify an $x \in{ }^{\omega} X$. I wins $G_{X}(A)$ if $x \in A$ (the "payoff" set), and otherwise $I I$ wins. A strategy is a function

\footnotetext{
${ }^{11}$ See Paul Larson's chapter for more details on determinacy.
} 
that tells a player what move to make given the sequence of previous moves. A winning strategy is a strategy such that if a player plays according to it he always wins no matter what his opponent plays. $A$ is determined if either $I$ or $I I$ has a winning strategy in $G_{X}(A)$.

David Gale and James Stewart [1953] initiated the study of these games and observed that if $A \subseteq{ }^{\omega} X$ is open (in the product topology) then $A$ is determined. The simple argument turned on how membership is secured at a finite stage, and a basic stratagem in the further investigations of determinacy would be the reduction to such "open games". Focusing on the basic case $X=\omega$ and noting that a strategy then can itself be construed as a real, Gale and Stewart showed by diagonalizing through all strategies that assuming $\mathrm{AC}$ there is an undetermined $A \subseteq{ }^{\omega} \omega$. Determinacy itself would come to be regarded as a regularity property, but there were basic difficulties from the beginning. Gale and Stewart asked whether all Borel sets of reals are determined, and in the decade that followed only sets very low in the Borel hierarchy were shown to be determined.

Infinitely long games involving reals had been considered as early as in the 1920s by mathematicians of the Polish school. With renewed interest in the subject in the $1950 \mathrm{~s}$, and with determinacy increasingly seen to be potent in its consequences, Jan Mycielski and Hugo Steinhaus [1962] proposed the following axiom, now known as the Axiom of Determinacy (AD):

\section{Every $A \subseteq{ }^{\omega} \omega$ is determined.}

With $\mathrm{AD}$ contradicting $\mathrm{AC}$ they proposed from the beginning that in the $\mathrm{ZFC}$ context the axiom should hold in some inner model. Solovay pointed out the natural candidate $L(\mathbb{R})$, the constructible closure of the reals $\mathbb{R}={ }^{\omega} \omega$, observing that if $\mathrm{AD}$ holds then $\mathrm{AD}^{L(\mathbb{R})}$, i.e. $\mathrm{AD}$ holds in $L(\mathbb{R})$. Further restricted hypotheses would soon be applied to the tasks at hand: Projective Determinacy (PD) asserts that every projective $A \subseteq \omega_{\omega}$ is determined; $\boldsymbol{\Sigma}_{n}^{1}$-determinacy, that every $\boldsymbol{\Sigma}_{n}^{1}$ set $A$ is determined; and so forth.

By 1964, games to specific purposes had been devised to show that for $A \subseteq$ ${ }^{\omega} \omega$ there is a closely related $B \subseteq{ }^{\omega} \omega$ (a continuous preimage) so that if $B$ is determined, then $A$ is Lebesgue measurable, and similarly for the Baire property and the perfect set property. Moreover, AD does imply a limited choice principle, that every countable set consisting of sets of reals has a choice function. Thus, the groundwork was laid for the reign of $\mathrm{AD}$ in $L(\mathbb{R})$ to enforce the regularity properties for all sets of reals there as well as a local choice principle, and unfettered uses of $\mathrm{AC}$ relegated to the universe at large.

In 1967 two results drew determinacy to the foreground of set theory, one about the transfinite and the other about definable sets of reals. Solovay established that $\mathrm{AD}$ implies that $\omega_{1}$ is measurable, injecting emerging large cardinal techniques into a novel setting without AC. David Blackwell [1967] provided a new proof via the determinacy of open games of a classical result of Kuratowski that the $\boldsymbol{\Pi}_{1}^{1}$ sets have the reduction property, one of several properties investigated in descriptive set theory for the projective sets. These results stimulated interest because of their 
immediacy and new approach to proof, that of devising a game and appealing to the existence of winning strategies to deduce a dichotomy. Martin in particular saw the potentialities at hand and soon made incisive contributions to investigations with and of determinacy. He initially made a simple but crucial observation based on the construal of strategies as reals that would have myriad applications: Under $\mathrm{AD}$ the filter over the Turing degrees generated by the cones is an ultrafilter.

Martin and Yiannis Moschovakis independently in 1968 extended the reduction property through the projective hierarchy by playing games and assuming PD, realizing a methodological goal of the classical descriptive set theorists by carrying out an inductive propagation. This was Martin's initial application of his ultrafilter on Turing cones, and the idea of ranking ordinal-valued functions via ultrafilters, so crucial in later arguments, first occurred here.

Already in 1964 Moschovakis had abstracted a property stronger and more intrinsic than reduction, the prewellordering property, from the classical analysis of $\Pi_{1}^{1}$ sets. A relation $\preceq$ is a prewellordering if it is a well-ordering except possibly that there could be distinct $x$ and $y$ such that $x \preceq y$ and $y \preceq x$. While a wellordering of a set $A$ corresponds to a bijection of $A$ into an ordinal, a prewellordering corresponds to a surjection onto an ordinal - a stratification of $A$ into well-ordered layers. A class $\boldsymbol{\Gamma}$ of sets of reals has the prewellordering property if for any $A \in$ $\boldsymbol{\Gamma}$ there is a prewellordering of $A$ such that both it and its complement are in $\boldsymbol{\Gamma}$ in a strong sense. This property supplanted the reduction property in the Martin-Moschovakis First Periodicity Theorem, which implied that under PD the prewellordering property holds periodically for the projective classes: $\boldsymbol{\Pi}_{1}^{1}, \boldsymbol{\Sigma}_{2}^{1}, \boldsymbol{\Pi}_{3}^{1}$, $\boldsymbol{\Sigma}_{4}^{1}, \ldots$

As for Solovay's result, he in fact established that under AD the closed unbounded filter $\mathcal{C}_{\omega_{1}}$ is an ultrafilter by using a game played with countable ordinals and simulating it with reals. Martin provided an alternate proof using his ultrafilter on Turing cones, and then Solovay in 1968 used Martin's result to establish that under $\mathrm{AD} \omega_{2}$ is measurable. With an apparent trend set, quite unexpected was the next advance. Martin in 1970 established that under AD the $\omega_{n}$ 's for $3 \leq n<\omega$ are all singular with cofinality $\omega_{2}$ ! This was a by-product of an incisive analysis of Martin's of $\boldsymbol{\Sigma}_{3}^{1}$ sets under AD.

Martin [1970] also reactivated the earlier project of securing more and more determinacy by establishing that if there is a measurable cardinal, then $\boldsymbol{\Pi}_{1}^{1}$ determinacy holds, or in refined terms, if $a^{\#}$ exists, then $\Pi_{1}^{1}(a)$-determinacy holds. The proof featured a remarkably simple reduction to an open game, based on indiscernibles and homogeneity properties, of form $G_{X}(A)$ for a set $X$ of ordinals. This ground-breaking proof served both to make plausible the possibility of getting $\mathrm{PD}$ from large cardinals as well as getting $\boldsymbol{\Delta}_{1}^{1}$-determinacy, Borel Determinacy, in ZFC - both directions to be met with complete success in later years.

The advances in the investigation of definable sets of reals with determinacy would be in terms of their analysis as projections of trees. For purposes of descriptive set theory, $T$ is a tree on $\omega \times \kappa$ iff (a) $T$ consists of pairs $\langle s, t\rangle$ where $s$ is a finite sequence drawn from $\omega$ and $t$ is a finite sequence drawn from $\kappa$ of the 
same length, and (b) if $\langle s, t\rangle \in T, s^{\prime}$ is an initial segment of $s$ and $t^{\prime}$ is a initial segment of $t$ of the same length, then also $\left\langle s^{\prime}, t^{\prime}\right\rangle \in T$. For such $T,[T]$ consists of pairs $\langle f, g\rangle$ corresponding to infinite branches, i.e. $f$ and $g$ are $\omega$-sequences such that for any finite initial segment $s$ of $f$ and finite initial segment $t$ of $g$ of the same length, $\langle s, t\rangle \in T$. In modern terms, $A \subseteq \omega_{\omega}$ is $\kappa$-Suslin iff there is a tree on $\omega \times \kappa$ such that $A=p[T]=\{f \mid \exists g(\langle f, g\rangle \in[T])\}$. [T] is a closed set in the space of $\langle f, g\rangle$ 's where $f: \omega \rightarrow \omega$ and $g: \omega \rightarrow \kappa$, and so otherwise complicated sets of reals, if shown to be $\kappa$-Suslin, are newly comprehended as projections of closed sets. The analytic $\left(\boldsymbol{\Sigma}_{1}^{1}\right)$ sets are exactly the $\omega$-Suslin sets. Shoenfield [1961] had established that every $\Sigma_{2}^{1}$ set is $\omega_{1}$-Suslin.

To address the classical issue of uniformization, Moschovakis [1971] abstracted from the proof of the classical, Kondô $\Pi_{1}^{1}$ Uniformization Theorem what would become the central structural concept in the investigation of definable sets of reals with determinacy. A scale on a set $A \subseteq \omega_{\omega}$ is an $\omega$-sequence of ordinal-valued functions on $A$ satisfying convergence and continuity properties, and a class $\boldsymbol{\Gamma}$ of sets of reals has the scale property if for any $A \in \boldsymbol{\Gamma}$ there is a scale on $A$ whose corresponding graph relations are in $\Gamma$ in a strong sense. Having a scale on $A$ corresponds to having $A=p[T]$ for a tree $T$ in such a way that, importantly, from $A$ is definable a member of $A$ through a minimization process ("choosing the honest leftmost branch").

In the early 1970s Moschovakis, Martin, and Alexander Kechris proceeded with scales to provide a detailed analysis of the projective sets under PD in terms of Borel sets and as projections of trees, based on the projective ordinals $\boldsymbol{\delta}_{n}^{1}=$ the supremum of the lengths of the $\boldsymbol{\Delta}_{n}^{1}$ prewellorderings. For example, the $\boldsymbol{\Sigma}_{2 n+2}^{1}$ sets are exactly the $\boldsymbol{\delta}_{2 n+1}^{1}$-Suslin sets. The projective ordinals themselves were subjected to considerable scrutiny, with penetrating work of Kunen particularly advancing the theory, and were found to be measurable and to satisfy strong partition properties. However, where exactly the $\boldsymbol{\delta}_{n}^{1}$ for $n \geq 5$ are in the aleph hierarchy would remain a mystery until the latter 1980s, when Steve Jackson [1988] in a tour de force settled the question with a deep analysis of the ultrafilters and partition properties involved. As an otherwise complete structure theory for projective sets was being worked out into the 1970s, Martin in 1974 (cf. [1975]) returned to a bedrock issue for the regularity properties and established in ZFC that $\boldsymbol{\Delta}_{1}^{1}$-determinacy, Borel Determinacy, holds.

\subsection{Elaborations}

Although large large cardinals were developed particularly to investigate the possibilities for elementary embeddings and were quickly seen to have a simple but elegant basic theory, what really intimated their potentialities were new forcing results in the 1970 s and 1980 s, especially from supercompactness, that established new relative consistencies, even of assertions low in the cumulative hierarchy. The earliest, orienting result along these lines addressed the Singular Cardinals Problem. The "Prikry-Silver" result provided the first instance of a failure of the 
Singular Cardinal Hypothesis by drawing together two results of independent significance, themselves crucial as methodological advances.

Karel Prikry in his 1968 Berkeley thesis (cf. his [1970]) had set out a simple but elegant notion of forcing that changed the cofinality of a measurable cardinal without collapsing any cardinals. With $U$ a normal $\kappa$-complete ultrafilter over $\kappa$, Prikry forcing for $U$ has as conditions $\langle p, A\rangle$ where $p$ is a finite subset of $\kappa$ and $A \in U$. For conditions $\langle p, A\rangle$ and $\langle q, B\rangle$, the first is stronger than the second if $p \supseteq q$ and $\alpha \in p-q$ implies $\alpha>\max (q)$ and $A \cup(p-q) \subseteq B$. A condition thus specifies a finite initial part of a new $\omega$-cofinalizing subset of $\kappa$, and further members are to be added on top from a set large in the sense of being in $U$. Applying a partition relation available for normal ultrafilters, Prikry established a focal property, that for any condition $\langle p, A\rangle$ and forcing statement, there is a $B \subseteq A$ such that $B \in U$ and $\langle p, B\rangle$ decides the statement, i.e. extending $p$ is unnecessary. Hence, e.g. the $\kappa$-completeness of $U$ implies that $V_{\kappa}$ remains unchanged in the forcing extension yet the cofinality of $\kappa$ now becomes $\omega$.

Prikry forcing may at first have seemed a curious possibility for singularization. However, that a Prikry generic sequence also generates the corresponding $U$ in simple fashion and also results from indiscernibles made them a central feature of measurability. Prikry forcing would be generalized in various directions and for a variety of purposes. With the capabilities made available for changing cofinalities, equi-consistency connections would eventually be established between large cardinals on the one hand and formulations in connection with the Singular Cardinals Problem on the other.

Silver in 1971 first established the relative consistency of having a measurable cardinal $\kappa$ satisfying $2^{\kappa}>\kappa^{+}$, a proposition that Kunen [1971b] had shown to be substantially stronger than just having measurability. Forcing over the model constructed by Silver with Prikry forcing yielded the first counterexample to the Singular Cardinals Hypothesis by providing a singular strong limit cardinal $\kappa$ satisfying $2^{\kappa}>\kappa^{+}$.

To establish his result, Silver provided a technique for extending elementary embeddings into generic extensions and thereby preserving large cardinal properties. To get at what is at issue, suppose that $j: V \rightarrow M$ is an elementary embedding, $P$ is a notion of forcing, and $G$ is $V$-generic for $P$. To extend (or "lift") $j$ to an elementary embedding for $V[G]$, the natural scheme would be to get an $M$-generic $G^{\prime}$ for $j(P)$ and extend $j$ to an elementary embedding from $V[G]$ into $M\left[G^{\prime}\right]$. But for this to work with the forcing terms, it would be necessary to enforce

$$
\forall p \in G\left(j(p) \in G^{\prime}\right) .
$$

For getting a measurable cardinal $\kappa$ satisfying $2^{\kappa}=\kappa^{++}$, Silver started with an elementary embedding as above with critical point $\kappa$ and devised a $P$ for adjoining $\kappa^{++}$Cohen subsets of $\kappa$. In order to establish a close connection between $P$ and $j(P)$ toward securing $(*)$, he took $P$ to be a uniform iteration of forcings to adjoin $\lambda^{++}$Cohen subsets of $\lambda$ for every inaccessible cardinal $\lambda$ up to and including $\kappa$. Then with the shift from $\kappa$ to $j(\kappa), j(P)$ can be considered a two-stage iteration 
of $P$ followed by a further iteration $Q$. Now with $G V$-generic for $P, G$ is also $M$-generic for $P$, and in $M[G]$ one should devise an $H M[G]$-generic for $Q$ such that the combined generic $G^{\prime}=G * H$ satisfies (*).

But how is this to be arranged? Silver was able to control the $j(p)$ 's for $p \in G$ by a single, (strong) master condition $q \in Q$, and build in $V[G]$ an $H M[G]$-generic over $Q$ with $q \in H$ to satisfy $(*)$. For getting both $q$ and $H$, he needed that $M$ be closed under arbitrary $\kappa^{++}$-sequences. Thus he established: If $\kappa$ is $\kappa^{++}$. supercompact, then there is a forcing extension in which $\kappa$ is measurable and $2^{\kappa}=$ $\kappa^{++} .12$ Silver's preparatory "reversed Easton" forcing with Easton support and master condition constructions of generic filters would become staple ingredients for the generic extension of elementary embeddings.

What about the use of very strong hypotheses in consistency results? A signal, 1972 result of Kunen (cf. [1978]) brought into play the strongest hypothesis to date for establishing a consistency result about the low levels of the cumulative hierarchy. Kunen [1970] had established that having a $\kappa$-complete $\kappa^{+}$-saturated ideal over a successor cardinal $\kappa$ has consistency strength substantially stronger than having a measurable cardinal. Kunen now showed: If $\kappa$ is huge, then there is forcing extension in which $\kappa=\omega_{1}$ and there is an $\aleph_{1}$-complete $\aleph_{2}$-saturated ideal over $\omega_{1}$. With a $j: V \rightarrow M$ with critical point $\kappa, \lambda=j(\kappa)$, and ${ }^{\lambda} M \subseteq M$ as given by the hugeness of $\kappa$, Kunen collapsed $\kappa$ to $\omega_{1}$ and followed it was a collapse of $\lambda$ to $\omega_{2}$ in such a way so as to be able to define a saturated ideal. Crucially, the first collapse was a "universal" collapse $P$ iteratively constructed so that the second collapse can be absorbed into $j(P)$ in a way consistent with $j$ applied to $P$, and this required ${ }^{\lambda} M \subseteq M$. Hence, a sufficient algebraic argument was contingent on a closure property for an elementary embedding, one plucked from the emerging large cardinal hierarchy. In the years to come, Kunen's argument would be elaborated and emended to become the main technique for getting various sorts of saturated ideals over accessible cardinals. As for the proposition that there is an $\aleph_{1}$-complete $\aleph_{2}$-saturated ideal over $\omega_{1}$ itself, Kunen's result set an initial high bar for the stalking of its consistency strength, but definitive work of the 1980s would show that far less than hugeness suffices.

William Mitchell would become a major developer of inner models for large cardinals, the first after Kunen. His Berkeley thesis (cf. [1972]), however, made important strides in a different direction. A $\kappa$-tree is special if it is the union of fewer than $\kappa$ antichains. Aronszajn's original construction of an Aronszajn tree actually provided a special tree. Analogously, a $\kappa$-Aronszajn tree is a $\kappa$-tree with no cofinal branches, i.e. a counterexample to the tree property for $\kappa$, and a generalization of Aronszajn's construction shows that if $\kappa$ is regular and $2^{\alpha} \leq \kappa$ for every $\alpha<\kappa$, then there is a special $\kappa^{+}$-Aronszajn tree. Mitchell showed: If there is a Mahlo cardinal, then there is a forcing extension making it $\omega_{2}$ in which there are no special $\omega_{2}$-Aronszajn trees. The forcing added Cohen reals to

\footnotetext{
${ }^{12}$ To mention an elegant coda, work of Woodin and Gitik in the 1980s showed that having a measurable cardinal satisfying $2^{\kappa}>\kappa^{+}$is equi-consistent with the proposition $\exists \kappa\left(o(\kappa)=\kappa^{++}\right)$, formulated below in the text.
} 
make the eventual $2^{\aleph_{0}}$ necessarily $\omega_{2}$, and had sophisticated features that could count as "countable support iteration" and "termspace forcing", important in later iterated forcing. Mitchell also observed that if there are no special $\omega_{2}$-Aronszajn trees, then $\omega_{2}$ is Mahlo in L. Hence we have the equi-consistency: Having no special $\omega_{2}$-Aronszajn trees is equi-consistent to having a Mahlo cardinal. Recall that a characterization of a cardinal $\kappa$ being weakly compact is being inaccessible and having the tree property (i.e. having no $\kappa$-Aronszajn trees at all). Mitchell's advisor Silver applied Mitchell's forcing argument and made a corresponding inner model observation to establish an equi-consistency result for the tree property at an accessible cardinal: $\omega_{2}$ having the tree property is equi-consistent to having a weakly compact cardinal.

After a brief excursion into category theory, Mitchell [1974] soon provided the first substantive extension of Kunen's inner model results and brought to prominence a new, delimitative, large cardinal hypothesis. For normal $\kappa$-complete ultrafilters $U$ and $U^{\prime}$ over $\kappa$, define the Mitchell order $U^{\prime} \triangleleft U$ iff $U^{\prime} \in \operatorname{Ult}(V, U)$, i.e. there is an $f: \kappa \rightarrow V$ representing $U^{\prime}$ in the ultrapower, so that $\{\alpha<\kappa \mid f(\alpha)$ is a normal $\alpha$-complete ultrafilter over $\alpha\} \in U$ and $\kappa$ is already a limit or measurable cardinals. $U \triangleleft U$ always fails, and generally, $\triangleleft$ is a well-founded relation by a version of Scott's argument that measurable cardinals contradict $V=L$. Consequently, to each $U$ can be recursively assigned a rank $o(U)=\sup \left\{o\left(U^{\prime}\right)+1 \mid\right.$ $\left.U^{\prime} \triangleleft U\right\}$, and to a cardinal $\kappa$, the supremum $o(\kappa)=\sup \{o(U)+1 \mid U$ is a normal $\kappa$-complete ultrafilter over $\kappa$ \}. By a cardinality argument, if $2^{\kappa}=\kappa^{+}$then $o(\kappa) \leq \kappa^{++}$.

The hypothesis $o(\kappa)=\delta$ provided an "order" of measurability calibrated by $\delta$, with larger $\delta$ corresponding to stronger assumptions on $\kappa$. For the investigation of these orders, Mitchell devised the concept of a coherent sequence of ultrafilters ("measures") and was able to establish canonicity results for inner models $L[\mathcal{U}]=$ " $\mathcal{U}$ is a coherent sequence of ultrafilters". A coherent sequence $\mathcal{U}$ is a doubly indexed system of normal $\alpha$-complete ultrafilters $\mathcal{U}(\alpha, \beta)$ over $\alpha$ such that $\mathcal{U}(\kappa, \beta) \triangleleft \mathcal{U}\left(\kappa, \beta^{\prime}\right)$ for $\beta<\beta^{\prime}$ at the $\kappa$ th level, and the earlier levels contain just enough ultrafilters necessary to represent these $\triangleleft$ relationships in the respective ultrapowers. (Technically, if $j: V \rightarrow \operatorname{Ult}\left(V, \mathcal{U}\left(\kappa, \beta^{\prime}\right)\right.$ ), then $j(\mathcal{U})\left\lceil\{(\alpha, \beta) \mid \alpha \leq \kappa\}=\mathcal{U}\left\lceil\left\{(\alpha, \beta) \mid \alpha<\kappa \vee\left(\alpha=\kappa \wedge \beta<\beta^{\prime}\right)\right\}\right.\right.$, i.e. $j(\mathcal{U})$ through $\kappa$ is exactly $\mathcal{U}$ "below" $\left(\kappa, \beta^{\prime}\right)$.)

Mitchell first affirmed that these $L[\mathcal{U}]$ 's are iterable in that arbitrary iterated ultrapowers via ultrafilters in $\mathcal{U}$ and its successive images are always well-founded. He then effected a comparison: $A n y L\left[\mathcal{U}_{1}\right]$ and $L\left[\mathcal{U}_{2}\right]$ have respective iterated ultrapowers $L\left[\mathcal{W}_{1}\right]$ and $L\left[\mathcal{W}_{2}\right]$ such that $\mathcal{W}_{1}$ is an initial segment of $\mathcal{W}_{2}$ or vice versa. This he achieved through a process of coiteration of least differences: At each stage, one finds the lexicographically least coordinate at which the current iterated ultrapowers of $L\left[\mathcal{U}_{1}\right]$ and $L\left[\mathcal{U}_{2}\right]$ differ and takes the respective ultrapowers by the differing ultrafilters; the difference is eliminated as ultrafilters never occur in their ultrapowers. With this coiteration, Mitchell established that in $L[\mathcal{U}]$ the only normal $\alpha$-complete ultrafilters over $\alpha$ for any $\alpha$ are those that occur in $\mathcal{U}$ and other 
propositions like GCH that showed these models to be $L$-like. Coiteration would henceforth be embedded in inner model theory, and with his models $L[\mathcal{U}]$ modeling $o(\kappa)=\delta$ for $\delta<\kappa^{++L[\mathcal{U}]}, \exists \kappa\left(o(\kappa)=\kappa^{++}\right)$would become the delimitative proposition of his analysis.

Much of the early formative work on strong large cardinal hypotheses and their integration into modern set theory through consistency results was carried out by Menachem Magidor, whose subsequent, broad-ranging initiatives have considerably advanced the entire subject. After completing his Hebrew University thesis in 1972 on supercompact cardinals, Magidor in the 1970s established a series of penetrating forcing consistency results involving strong hypotheses. In 1972-3 (cf. [1976]) he illuminated the "identity crisis" issue of whether supercompactness and strong compactness are distinct concepts by establishing: (1) It is consistent that the least supercompact cardinal is also the least strong compact cardinal, and (2) It is consistent that the least strong compact cardinal is the least measurable cardinal (and so, much smaller than the least supercompact cardinal). The proofs showed how changing many cofinalities with Prikry forcing to destroy measurable cardinals can be integrated into arguments about extending elementary embeddings.

In 1973 Magidor (cf. [1977a]) showed how with strong hypotheses the least limit cardinal $\aleph_{\omega}$ can violate the Singular Cardinals Hypothesis: If $\kappa$ is supercompact, there is a forcing extension in which $\kappa$ is $\aleph_{\omega}$ as a strong limit cardinal yet $2^{\aleph_{\omega}}>$ $\aleph_{\omega+1}$. The strong elementary embedding hypothesis allowed for an elaborated Prikry forcing interspersed with Levy collapses. With this first net thrown out, it would be one of the great elaborations of method that equi-consistency results would eventually be achieved with weaker hypotheses for the violation of $\mathrm{SCH}$, and already at $\aleph_{\omega}$.

\subsection{Silver's theorem and covering}

In mid-1974 Silver [1975] established that if $\kappa$ is a singular cardinal with $\mathrm{cf}(\kappa)>\omega$ and $2^{\lambda}=\lambda^{+}$for $\lambda<\kappa$, then $2^{\kappa}=\kappa^{+}$. This was a dramatic event and would stimulate dramatic developments. There had been precious little in the way of results provable in ZFC about cardinal arithmetic, and in the early ruminations about the Singular Cardinals Problem it was quite unforeseen that the power of a singular cardinal can be so constrained. An analogous preservation result had been observed by Scott for measurable cardinals, and telling was that Silver used large-cardinal ideas connected with generic ultrapowers.

Silver's result spurred broad-ranging investigations both into the combinatorics and avenue of proof and into larger, structural implications. The basis of his argument was a ranking of ordinal-valued functions on $\operatorname{cf}(\kappa)$. Let $\left\langle\gamma_{\alpha} \mid \alpha<\operatorname{cf}(\kappa)\right\rangle$ be a sequence of ordinals unbounded in $\kappa$ and for $\alpha<\operatorname{cf}(\kappa)$ let $\tau_{\alpha}: P\left(\gamma_{\alpha}\right) \rightarrow 2^{\gamma_{\alpha}}$ be a bijection. For $X \subseteq \kappa$ let $f_{X}$ on $\operatorname{cf}(\kappa)$ be defined by: $f_{X}(\alpha)=\tau_{\alpha}\left(X \cap \gamma_{\alpha}\right)$, noting that $X_{1} \neq X_{2}$ implies $f_{X_{1}}$ and $f_{X_{2}}$ differ for sufficiently large $\alpha$. Then $2^{\kappa}$ is mirrored through these eventually different functions, which one can work to order 
according to an ideal over the uncountable $\operatorname{cf}(\kappa)$. The combinatorial possibilities of such rankings led to a series of limitative results on the powers of singular cardinals of uncountable cofinality, starting with the results of Fred Galvin and Hajnal [1975], of which the paradigmatic example is that if $\aleph_{\omega_{1}}$ is a strong limit cardinal, then $2^{\aleph_{\omega_{1}}}<\aleph_{\left(2^{\aleph_{1}}\right)+}$.

In the wake of Silver's proof, Thomas Jech and Prikry [1976] defined a $\kappa$ complete ideal over $\kappa$ to be precipitous iff the corresponding generic ultrapower à la Solovay is well-founded. They thus put the focus on a structural property of saturated ideals that Silver had simulated to such good effect. Jech and Prikry pointed out that a proof of Kunen's for saturated ideals using iterated ultrapowers can be tailored to show: If there is a precipitous ideal over $\kappa$, then $\kappa$ is measurable in an inner model. Then Mitchell observed (cf. [Jech et al., 1980]): If a measurable cardinal is Levy collapsed to $\omega_{1}$, then a normal ultrafilter generates a precipitous ideal over $\omega_{1}$ in the extension. Hence, a first equi-consistency result was achieved for measurability and $\omega_{1}$ in ZFC. With combinatorial characterizations of precipitousness soon in place, well-foundedness as thus modulated by forcing became a basic ingredient in a large-scale investigation of strong properties tailored to ideals and generic elementary embeddings.

The most dramatic and penetrating development from Silver's Theorem was Jensen's work on "covering" for $L$ and its first extensions, the most prominent advances of the 1970s in set theory. Jensen had found Silver's result a "shocking discovery", and was stimulated to intense activity. By the end of 1974 he had made prodigious progress, solving the Singular Cardinals Problem in the absence of $0^{\#}$ in three manuscripts, "Marginalia to a Theorem of Silver" and its two sequels. The culminating result featured an elegant and focal formulation of intuitive immediacy, the Covering Theorem (or "Lemma") for L: If $0^{\#}$ does not exist, then for any uncountable set $X$ of ordinals there is a $Y \in L$ with $|Y|=|X|$ such that $Y \supseteq X$. (The first published account was given in [Devlin and Jensen, 1975]; without the "uncountable" there would be a counterexample using "Namba forcing".) This covering property expresses a global affinity between $V$ and $L$, and its contrapositive provides a surprisingly simple condition sufficient for the existence of $0^{\#}$ and the ensuing indiscernible generation of $L$. As such, Jensen's theorem would find wide applications for implicating $0^{\#}$ and would provide a new initiative in inner model theory for encompassing stronger hypotheses.

The Covering Theorem gave the essence of Jensen's argument that in the absence of $0^{\#}$ the Singular Cardinals Hypotheses holds: Suppose that $\kappa$ is singular and for reckoning with the powers of smaller cardinals consider $\lambda=\sup \left\{2^{\mu} \mid\right.$ $\mu<\kappa\}$. If there is a $\nu<\kappa$ such that $\lambda=2^{\nu}$, then the functions $f_{X}$ defined as above adapted to the present situation satisfy $f_{X}: \operatorname{cf}(\kappa) \rightarrow 2^{\nu}$, and so $\lambda \leq 2^{\kappa} \leq\left(2^{\nu}\right)^{\operatorname{cf}(\kappa)} \leq \lambda$. If on the other hand $\lambda$ is the strict supremum of increasing $2^{\mu}$ 's, then $\operatorname{cf}(\lambda)=\operatorname{cf}(\kappa)$ and so the Zermelo-Konnig inequality would dictate the least possibility for $2^{\kappa}$ to be $\lambda^{+}$. However, if for any $X \subseteq \kappa$ the range of $f_{X}$ is covered by a $Y \subseteq \lambda$ with $Y \in L$ of cardinality $\operatorname{cf}(\kappa) \cdot \aleph_{1}$, then: there are $2^{\operatorname{cf}(\kappa) \cdot \aleph_{1}}$ subsets of each such $Y$ and by the GCH in $L$, at most $\left|\lambda^{+L}\right|$ such $Y$. Hence, we 
would have $2^{\kappa} \leq 2^{\operatorname{cf}(\kappa) \cdot \aleph_{1}} \cdot\left|\lambda^{+L}\right| \leq \lambda^{+}$.

The Covering Theorem also provided another dividend that would grow in separate significance as having the weak covering property: Assume that $0^{\#}$ does not exist. If $\kappa$ is singular, then $\kappa^{+L}=\kappa^{+}$. If to the contrary $\kappa^{+L}<\kappa^{+}$, then $\operatorname{cf}\left(\kappa^{+L}\right)<\kappa$. Let $X \subseteq \kappa^{+L}$ be unbounded so that $|X|<\kappa$ and let $Y \in L$ cover $X$ with $|Y|=|X| \cdot \aleph_{1}$. But then, the order-type of $Y$ would be less than $\kappa$, contradicting the regularity of $\kappa^{+L}$ in $L$.

A crucial consequence of weak covering is that in the absence of $0^{\#}, \square_{\kappa}$ holds for singular $\kappa$, since a $\square_{\kappa}$ sequence in the sense of $L$ is then a $\square_{\kappa}$ sequence in $V$. The weak covering property would itself become pivotal in the study of inner models corresponding to stronger and stronger hypotheses, and the failure of $\square_{\kappa}$ for singular $\kappa$ would become a delimitative proposition. Solovay (cf. [Solovay et al., 1978]) had already established an upper bound on consistency by showing in the early 1970s that if $\kappa$ is $\lambda^{+}$-supercompact and $\lambda \geq \kappa$, then $\square_{\lambda}$ fails.

Jensen's ingenious proof of the Covering Theorem for $L$ proceeded by taking a counterexample $X$ to covering with $\tau=\sup (X)$ and $|X|$ minimal; getting a certain $\Sigma_{1}$-elementary $j: L_{\gamma} \rightarrow L_{\tau}$ which contains $X$ in its range through a Skolem hull construction so that $|\gamma|=|X|$ and, as $X$ cannot be covered, $\gamma$ is a cardinal in $L$; and extending $j$ to an elementary embedding from $L$ into $L$, so that $0^{\#}$ exists. The procedure for extending $j$ up to some large $L_{\delta}$ was to consider a directed system of embeddings of structures generated by $\xi \cup p$ for some $\xi<\gamma$ and $p$ a finite subset of $L_{\delta}$, the transitized components of the system all being members of $L_{\gamma}$ as $\gamma$ is a cardinal in $L$, and to consider the corresponding directed system consisting of the $j$ images. The choice of $\gamma$ insured that the new directed system is also well-founded, and so isomorphic to some $L_{\zeta}$.

How can the proof of the Covering Theorem be adapted to establish a stronger result? The only possibility was to consider a larger inner model $M$ and to establish that $M$ has the covering property: for any uncountable set $X$ of ordinals there is a $Y \in M$ with $|Y|=|X|$ such that $Y \supseteq X$. In groundbreaking work for inner model theory, Solovay in the early 1970s had developed a fine structure theory for inner models of measurability. Whilst a research student at Oxford University Anthony Dodd worked through this theory, and in early 1976 he and Jensen laid out the main ideas for extending the Covering Theorem to a new inner model, now known as the Dodd-Jensen core model, denoted $K^{\mathrm{DJ}}$. (See their [1981] and the full exposition [Dodd, 1982].)

If $\langle L[U], \in, U\rangle$ is an inner model of measurability, say the $\kappa$-model, then there is a generic extension in which covering fails: If $G$ is Prikry generic for $U$ over $L[U]$, then $G$ cannot be covered by any set in $L[U]$ of cardinality less than $\kappa$. Drawing back, there remains the possibility of "iterating out" the measurable cardinal: If $\langle L[U], \in, U\rangle$ is the $\kappa$-model, then $\langle L[W], \in, W\rangle$ is the $\lambda$-model for some $\lambda>\kappa$ exactly when it is an iterate of $\langle L[U], \in, U\rangle$, in which case $L[W] \subseteq L[U]$, $V_{\kappa} \cap L[U]=V_{\kappa} \cap L[W]$, and $U \notin L[W]$. Thus, if $\left\langle L\left[U_{\alpha}\right]\right| \alpha \in$ On $\rangle$ enumerates the inner models of measurability, then starting with any one of them, the process of iterating it through the ordinals converges to a proper class $\bigcap_{\alpha} L\left[U_{\alpha}\right]$ which 
has no inner models of measurability, with the stabilizing feature that for any $\gamma, V_{\gamma} \cap \bigcap_{\alpha} L\left[U_{\alpha}\right]=V_{\gamma} \cap L\left[U_{\beta}\right]$ for sufficiently large $\beta$. Assuming that there are inner models of measurability, $K^{\mathrm{DJ}}$ is in fact characterizable as this residue class. Aspiring to this, but without making any such assumption, Dodd and Jensen provided a formulation of $K^{\mathrm{DJ}}$ in $\mathrm{ZFC}$.

$K^{\text {DJ }}$ was the first inner model of ZFC since Gödel's $L$ developed using distinctly new generating principles. Dodd and Jensen's approach was to take $K^{\mathrm{DJ}}$ as the union of $L$ together with "mice". Loosely speaking, a mouse is a set $L_{\alpha}[U \mid$ such that

$$
\left\langle L_{\alpha}[U], \in, U\right\rangle \models U \text { is a normal ultrafilter over } \kappa
$$

satisfying: (i) there is a subset of $\kappa$ in $L_{\alpha+1}[U]-L_{\alpha}[U]$, so that $U$ is on the verge of not being an ultrafilter; (ii) $\left\langle L_{\alpha}[U], \in, U\right\rangle$ is iterable in that all the iterated ultrapowers are well-founded, and (iii) fine structure conditions about a projectum below $\kappa$ leading to (i). Mice can be compared by taking iterated ultrapowers, so that there is a natural prewellordering of mice, and moreover, crucial elements about $L$ can be lifted to the new situation because there is a generalization of condensation: $\Sigma_{1}$-elementary substructures of mice, when transitized, are again mice. This led to $K^{\mathrm{DJ}}=\mathrm{GCH}$, and that $K^{\mathrm{DJ}}$ in the sense of $K^{\mathrm{DJ}}$ is again $K^{\mathrm{DJ}}$.

Mice generate indiscernibles through iteration, and so if $0^{\#}$ does not exist, then $K^{\mathrm{DJ}}=L$; if $0^{\#}$ exists but $0^{\# \#}$ does not, then $K^{\mathrm{DJ}}=L\left[0^{\#}\right]$; and this continues through the transfinite by coding sequences of sharps. On the other hand, $K^{\text {DJ }}$ has no simple constructive analysis from below and is rather like a maximal inner model on the brink of measurability: Its own "sharp", that there is an elementary embedding $j: K^{\mathrm{DJ}} \rightarrow K^{\mathrm{DJ}}$, is equivalent to the existence of an inner model of measurability. Indeed, this was Dodd and Jensen's primary motivation for the formulation of $K^{\mathrm{DJ}}$. They used it in place of the elementary embedding characterization of the existence of $0^{\#}$, together with the $L$-like properties of $K^{\mathrm{DJ}}$, to establish (cf. [1982a]) the Covering Theorem for $K^{\mathrm{DJ}}$ : If there is no inner model of measurability, then $K^{\mathrm{DJ}}$ has the covering property. This has the attendant consequences for the Singular Cardinals Problem. Moreover, Dodd and Jensen were able to establish a covering result for inner models of measurability that accommodates Prikry forcing. Solovay had devised a set of integers $0^{\dagger}$ ("zero dagger"), analogous to $0^{\#}$, such that $0^{\dagger}$ exists exactly when for some $\kappa$-model $L[U]$ there is an elementary embedding $j: L[U] \rightarrow L[U]$ with critical point above $\kappa$. Dodd and Jensen (cf. [1982b]) established: If $0^{\dagger}$ does not exist yet there is an inner model of measurability, then for the $\kappa$-model $L[U]$ with $\kappa$ least, either (a) $L[U]$ has the covering property, or (b) there is a Prikry generic $G$ for $U$ over $L[U]$ such that $L[U][G]$ has the covering property. Prikry forcing provides the only counterexample to covering! Hence, the inner models thus far considered were also "core models", models on the brink so that the lack of covering leads to the next large cardinal hypothesis.

In the light of the Dodd-Jensen work, Mitchell in the later 1970s (cf. [1984; $1985]$ ) developed the core model $K[\mathcal{U}]$ for coherent sequences $\mathcal{U}$ of ultrafilters, which corresponds to his $L[\mathcal{U}]$ as $K^{\mathrm{DJ}}$ does to $L[U]$. The mice are now sets of 
form $J_{\alpha}[W]$ with iterability and fine structure properties, where $W$ is an ultrafilter sequence with $\mathcal{U}$ as an initial segment. Under the assumption that there is no inner model satisfying $\exists \kappa\left(o(\kappa)=\kappa^{++}\right)$, Mitchell established the weak covering property for $K[\mathcal{U}]$, i.e. that $\left(\kappa^{+}\right)^{K[\mathcal{U}]}=\kappa^{+}$for singular $\kappa$. With this he showed that several propositions have at least the consistency strength of $\exists \kappa\left(o(\kappa)=\kappa^{++}\right)$. One such proposition was that there is an $\aleph_{1}$-complete $\aleph_{2}$-saturated ideal over $\omega_{1}$, establishing a new lower bound in consistency strength for Kunen's consistency result from a huge cardinal. Mitchell [1985] established, generalizing the DoddJensen result with Prikry generic sets, a full covering theorem for $K[\mathcal{U}]$ cast in terms of coherent systems of indiscernibles and drew further conclusions about singular cardinals.

\subsection{Forcing consistency results through the 1970s}

Through the 1970s a wide range of variegated forcing consistency results were established at a new level of sophistication that clarified relationships among combinatorial propositions and principles and often drew in large cardinal hypotheses and stimulated the development of method, especially in iterated forcing. A conspicuous series of results resolved questions of larger mathematics (Whitehead's Problem, Borel's Conjecture, Kaplansky's Conjecture, the Normal Moore Space Problem) in terms of relative consistency and set-theoretic principles, newly affirming the efficacy and adjudicatory character of set theory. In what follows, we mention these results in turn as we continue to pursue the larger longitudinal themes, necessarily saying less and less about matters of increasing complexity.

In 1974 Magidor [1978] made a basic contribution to the theory of changing cofinalities, the first after Prikry. Magidor established: If a measurable cardinal $\kappa$ is of Mitchell order $o(\kappa) \geq \lambda$ for a regular $\lambda<\kappa$, then there is a forcing extension preserving cardinals in which $\operatorname{cf}(\kappa)=\lambda$. Generalizing Prikry forcing, Magidor's conditions consisted of a finite sequence of ordinals and a sequence of sets drawn from normal ultrafilters in the Mitchell order, the sets providing for the possible ways of filling out the sequence. Like Prikry's forcing, Magidor's may at first have seemed a curious possibility for a new singularization. However, one of the discernments of Mitchell's core model for coherent sequences of measures is that, remarkably [1987]: If a regular cardinal $\kappa$ in $V$ satisfies $\omega<\operatorname{cf}(\kappa)<\kappa$ in a generic extension, then $V$ has an inner model in which $o(\kappa)$ is at least that cofinality. Thus, the capability of changing cofinalities was exactly gauged; "Prikry-Magidor" generic sets as sequences of indiscernibles would become a basic component of Mitchell's covering work.

The most salient result of Magidor's of this period were one that provided counterweight to Silver's result for the least limit cardinal $\aleph_{\omega}$. Magidor [1977b] showed: If $\kappa$ is a huge cardinal, then there is a forcing extension in which $\kappa=\aleph_{\omega}$, $2^{\aleph_{n}}=\aleph_{n+1}$ for $n \in \omega$, yet $2^{\aleph_{\omega}}>\aleph_{\omega+1}$. This was the first time after Kunen's saturated ideal result (cf. [1978]) that the strength of a huge cardinal was invoked; with it, the forcing conditions from Magidor's earlier SCH argument [1977a] could 
be made more liberal in order to ensure that GCH held below $\aleph_{\omega}$.

With respect to the Jech-Prikry-Mitchell equi-consistency of measurability and precipitousness, Magidor (cf. [Jech et al., 1980]) showed that absorptive properties of the Levy collapse of a measurable cardinal to $\omega_{1}$ can be exploited by subsequently "shooting" closed unbounded subsets of $\omega_{1}$ through stationary sets to get: If there is a measurable cardinal $\kappa$, then there is a forcing extension in which $\kappa=\omega_{1}$ and $\mathrm{NS}_{\omega_{1}}$ is precipitous. Thus a basic, definable ideal can be precipitous, and this naturally became a principal point of departure for the investigation of ideals.

The move of Saharon Shelah into set theory in the early 1970s brought in a new and exciting sense of personal initiative that swelled into an enhanced purposiveness across the subject, both through his solutions of major outstanding problems as well as through his development of new structural frameworks. A phenomenal mathematician, Shelah from his 1969 Hebrew University thesis on has worked in model theory and eventually infused it with a transformative, abstract classification theory for models. In both model theory and set theory he has remained eminent and has produced results at a furious pace, with nearly 1000 items currently in his bibliography (his papers are currently archived at http://shelah.logic.at/).

In set theory Shelah was initially stimulated by specific problems. He typically makes a direct, frontal attack, bringing to bear extraordinary powers of concentration, a remarkable ability for sustained effort, an enormous arsenal of accumulated techniques, and a fine, quick memory. When he is successful on the larger problems, it is often as if a resilient, broad-based edifice has been erected, the traditional serial constraints loosened in favor of a wide, fluid flow of ideas and the final result almost incidental to the larger structure. What has been achieved is more than a just succinctly stated theorem but rather the erection of a whole network of robust arguments.

Shelah's written accounts have acquired a certain notoriety that in large part has to do with his insistence that his edifices be regarded as autonomous conceptual constructions. Their life is to be captured in the most general forms, and this entails the introduction of many parameters. Often, the network of arguments is articulated by complicated combinatorial principles and transient hypotheses, and the forward directions of the flow are rendered as elaborate transfinite inductions carrying along many side conditions. The ostensible goal of the construction, that succinctly stated result that is to encapsulate it, is often lost in a swirl of conclusions.

Shelah's first and very conspicuous advance in set theory was his 1973, definitive results on Whitehead's Problem in abelian group theory: Is every Whitehead group, i.e. an abelian group $G$ satisfying $\operatorname{Ext}^{1}(G, \mathbb{Z})=0$, free? Shelah [1974], established that $V=L$ implies that this is so, and that Martin's Axiom implies that there is a counterexample. Shelah thus established for the first time that a strong purely algebraic statement is undecidable in ZFC. With his $L$ result specifically based on diamond-type principles, Shelah brought them into prominence with his further work on them, which were his first incursions into iterated forcing. By the 
late 1970s his increasing understanding of and work in iterated forcing would put a firm spine on much of the variegated forcing arguments about the continuum.

With an innovative argument pivotal for iterated forcing, Richard Laver [1976] established the consistency of Borel's Conjecture: Every set of reals of strong measure zero is countable. $\mathrm{CH}$ had provided a counterexample, and Laver established the consistency with $2^{\aleph_{0}}=\aleph_{2}$. His argument featured the adjunction of what are now called Laver reals in the first clearly set out countable support iteration, i.e. an iteration with non-trivial local conditions allowed only at countably many coordinates. The earlier Solovay-Tennenbaum argument for the consistency of MA had relied on finite support, and the Mitchell argument about Aronszajn trees, an involved countable support with a "termspace" forcing. Laver's work showed that countable support iteration is both manageable and efficacious for preserving certain framing properties of the continuum to establish the consistency of propositions with $2^{\aleph_{0}}=\aleph_{2}$. Interestingly, a trade-off would develop however: while finite support iterations put all cardinals $\geq \aleph_{2}$ on an equal footing with respect to the continuum, countable support iterations restricted the continuum to be at most $\aleph_{2}$. With a range of new generic reals coming into play with the widening investigation of the continuum, James Baumgartner [1983] formulated a property common to the corresponding partial orders, Axiom A, which in particular ensured the preservation of $\omega_{1}$. He showed that the countable support iteration of Axiom A forcings is Axiom A, thereby uniformizing the iterative adjunction of the known generic reals.

All this would retrospectively have a precursory air, as Shelah soon established a general, subsuming framework. Analyzing Jensen's consistency argument for SH + $\mathrm{CH}$ and coming to grips with forcing names in iterated forcing, Shelah came to the concept of proper forcing as a general property that preserves $\omega_{1}$ and is preserved in countable support iterations. The instrumental formulation of properness is given in an appropriately broad setting:

First, for a regular cardinal $\lambda$, let $H(\lambda)=\{x|| \operatorname{tc}(\{x\}) \mid<\lambda\}$, the sets hereditarily of cardinality less than $\lambda$. The $H(\lambda)$ 's provide another cumulative hierarchy for $V$, one stratified into layers that each satisfy Replacement. Whereas the $V_{\alpha}$ 's for limit $\alpha$ satisfy every ZFC axiom except possibly Replacement, the $H(\lambda)$ 's satisfy every ZFC axiom except possibly Power Set. A partial order $\langle P,<\rangle$ is proper if for any regular $\lambda>2^{|P|}$ and countable $M \prec H(\lambda)$ with $P \in M$, every $p \in P \cap M$ has a $q \leq p$ such that $q \Vdash \dot{G} \cap M$ is $M$-generic. (Here, $\dot{G}$ a canonical name for a generic filter with respect to $P$, and $q$ forcing this genericity assertion has various combinatorial equivalents.)

A general articulation of how all countable approximations are to have generic filters has been achieved, and its presentation under countable support iterations exhibited the efficacy of this remarkable move to a new plateau. Shelah soon devised variants and augmentations, and in a timely monograph Proper Forcing [1982] revamped forcing for combinatorics and the continuum with systemic proofs of new and old results. As later presented in Shelah's mature book [1998] proper forcing has become a staple part of the methods of modern set theory, with its 
applications wide-ranging and the development of its extended theory a fount of research.

In light of Shelah's work and Martin's Axiom, Baumgartner in the early 1980s established the consistency of a new encompassing forcing axiom, the Proper Forcing Axiom (PFA): For any proper partial order $P$ and collection $\mathcal{D}$ of $\aleph_{1}$ dense subsets of $P$, there is a filter $G \subseteq P$ meeting every member of $\mathcal{D}$. Unlike MA, the consistency of PFA required large cardinal strength and moreover could not be achieved by iteratively taking care of the partial orders at issue, as new proper partial orders occur arbitrarily high in the cumulative hierarchy. Baumgartner established: If there is a supercompact cardinal $\kappa$, then there is a forcing extension in which $\kappa=\omega_{2}$ and PFA holds. In an early appeal to the full global reflection properties available at a supercompact cardinal Baumgartner iteratively took care of the emerging proper partial orders along a special diamond-like sequence that anticipates all possibilities. Laver [1978] had formulated such a sequence, the "Laver diamond", toward establishing what has become a useful result for forcing theory; in a forcing extension he made a supercompact cardinal "indestructible" by any further forcing from a substantial, useful class of forcings. PFA became a widely applied forcing axiom, showcasing Shelah's concept, but beyond that, it would itself become a pivotal hypothesis in the large cardinal context.

\section{NEW EXPANSION}

\subsection{Into the $1980 \mathrm{~s}$}

The 1980s featured a new and elaborating expansion in set theory significantly beyond the successes, already remarkable, of the previous decade. There were new methods and results of course, but more than that there were successful maximizations in several directions - definitive and evidently optimal resultsand successful articulations at the interstices-new concepts and refinements that filled out the earlier explorations. Our narrative now becomes even more episodic in increasingly inverse relation to the broad-ranging and penetrating developments.

In 1977 Lon Radin toward his Berkeley thesis developed (cf. [1982]) an ultimate generalization of the Prikry and Magidor forcings for changing cofinalities, a generalization which could in fact adjoin a closed unbounded subset, consisting of formerly regular cardinals, to a large cardinal $\kappa$ while maintaining its regularity and further substantive properties. As graduate students at Berkeley, Hugh Woodin and Matthew Foreman saw the possibilities abounding in Radin forcing. While an undergraduate at Caltech, Woodin did groundbreaking work (cf. [Dales and Woodin, 1987]), built on by Solovay, on the consistency of Kaplansky's Conjecture (Is every homomorphism on the Banach algebra of continuous functions on the unit interval continuous?) and now with Radin forcing in hand Woodin would produce his first series of remarkable results. By 1979 Foreman and Woodin (cf. their [1991]) had the essentials for establishing: If there is a supercompact cardinal $\kappa$, then there is forcing extension in which $V_{\kappa}$ as a model of ZFC satisfies 
that GCH fails everywhere, i.e. $2^{\lambda}>\lambda$ for every $\lambda$. This conspicuously subsumed the early Magidor result getting $\aleph_{\omega}$ a strong limit yet $2^{\aleph_{\omega}}>\aleph_{\omega+1}$ and put Radin forcing on the map for establishing global consistency results.

Shelah soon established two re-orienting results about powers of singular cardinals. Having come somewhat late into the game after Silver's Theorem, Shelah had nonetheless extended some of the limitative results about such powers, even to singular $\kappa$ such that $\aleph_{\kappa}=\kappa$. Shelah [1983] subsequently established: If there is a supercompact cardinal $\kappa$ and $\alpha$ is a countable ordinal, then there is a forcing extension in which $\kappa$ is $\aleph_{\omega}$ as a strong limit cardinal yet $2^{\aleph_{\omega}}=\aleph_{\alpha+1}$. He thus extended Magidor's result by showing that the power of $\aleph_{\omega}$ can be made arbitrarily large below $\aleph_{\omega_{1}}$. In 1980 Shelah established (cf. his book [1982]) the general result that for any limit ordinal $\delta, \aleph_{\delta}^{\mathrm{cf}(\delta)}<\aleph_{\left(|\delta|^{\mathrm{cf}(\delta)}\right)^{+}}$, so that in particular if $\aleph_{\omega}$ is a strong limit cardinal, then $2^{\aleph_{\omega}}<\aleph_{\left(2^{\left.\aleph_{0}\right)^{+}}\right.}$. Shelah thus brought countable cofinality, the one cofinality unattended to by Silver's Theorem, into the scheme of things by establishing a bound in ZFC analogous to the Galvin-Hajnal bound for uncountable cofinalities. Shelah's argument, based on the possible cofinalities of "reduced products" of a cofinal subset of $\aleph_{\delta}$, would evolve into a generally applicable method by the late 1980's, the remarkable pcf theory.

Mitchell [1978] made a new breakthrough for the inner model theory of large large cardinals by developing such a model for "hypermeasurable cardinals", e.g. a measurable cardinal $\kappa$ such that for some normal ultrafilter $U$ over $\kappa, P(P(\kappa)) \subseteq$ $\operatorname{Ult}(V, U)$, so that every ultrafilter over $\kappa$ is in the ultrapower. This at least captured a substantial consequence of the $2^{\kappa}$-supercompactness of $\kappa$, and so engendered the hope of getting $L$-like inner models for such strong hypotheses. Supercompactness, while increasingly relied on in relative consistency results owing to its reflection properties, was out of reach, but the Mitchell result suggested an appropriate weakening: A cardinal $\kappa$ is $\alpha$-strong iff there is an elementary embedding $j: V \rightarrow M$ for some inner model $M$, with critical point $\kappa$ and $\alpha<j(\kappa)$ such that $V_{\alpha} \subseteq M$. (One can alternately require that the $\alpha$ th iterated power set $P^{\alpha}(\kappa)$ be a subset of $M$, which varies the definition only for small $\alpha$ like $\alpha=2$ but makes the definition more germane for them.) $\kappa$ is strong iff it is $\alpha$-strong for every $\alpha$.

Dodd and Jensen (cf. [Dodd, 1982]) soon simplified Mitchell's presentation in what turned out to be a basic methodological advance for the development of inner model theory. While introducing certain redundancies, they formulated a general way of analyzing an elementary embedding in terms of extenders. The idea, anticipated in Jensen's proof of the Covering Theorem, is that elementary embeddings between inner models can be approximated arbitrarily closely as direct limits of ultrapowers with concrete features reminiscent of iterated ultrapowers.

Suppose that $N$ and $M$ are inner models of ZFC, $j: N \rightarrow M$ is elementary with a critical point $\kappa$, and $\beta>\kappa$. Let $\zeta \geq \kappa$ be the least ordinal satisfying $\beta \leq j(\zeta)$; the simple ("short") case is $\zeta=\kappa$, and the general case is for the study of stronger hypotheses. For each finite subset $a$ of $\beta$, define $E_{a}$ by: 


$$
X \in E_{a} \quad \text { iff } \quad X \in P\left([\zeta]^{|a|}\right) \cap N \wedge a \in j(X) .
$$

This is another version of the idea of generating ultrafilters from embeddings. $E_{a}$

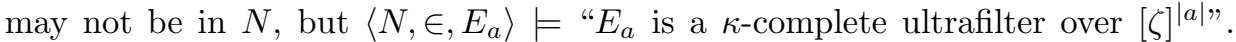
The $(\kappa, \beta)$-extender derived from $j$ is $E=\left\langle E_{a}\right| a$ is a finite subset of $\left.\beta\right\rangle$.

For each finite subset $a$ of $\beta, \operatorname{Ult}\left(N, E_{a}\right)$ is seen to be elementarily embeddable into $M$, so that in particular $\operatorname{Ult}\left(N, E_{a}\right)$ is well-founded and hence identified with its transitive collapse, say $M_{a}$. Next, for $a \subseteq b$ both finite subsets of $\beta$, corresponding to how members of $a$ sit in $b$ there is a natural elementary embedding $i_{a b}: M_{a} \rightarrow M_{b}$. Finally,

$$
\left.\left\langle\left\langle M_{a}\right| a \text { is a finite subset of } \beta\right\rangle,\left\langle i_{a b} \mid a \subseteq b\right\rangle\right\rangle
$$

is seen to be a directed system of structures with commutative embeddings, so stipulate that $\left\langle M_{E}, \in_{E}\right\rangle$ is the direct limit, and let $j_{E}: N \rightarrow M_{E}$ be the corresponding elementary embedding. We thus have the extender ultrapower of $N$ by $E$ as a direct limit of ultrapowers. The crucial point is that the direct limit construction ensures that $j_{E}$ and $M_{E}$ approximate $j$ and $M$ "up to $\beta$ ", e.g. if $\left|V_{\alpha}\right|^{M} \leq \beta$, then $\left|V_{\alpha}\right|^{M}=\left|V_{\alpha}\right|^{M_{E}}$, i.e. the cumulative hierarchies of $M$ and $M_{E}$ agree up to $\alpha$. Having formulated extenders derived from an embedding, a $(\kappa, \beta)$-extender is a sequence $E=\left\langle E_{a}\right| a$ is a finite subset of $\left.\beta\right\rangle$ that satisfies various abstracted properties that enable the above construction.

In a manuscript circulated in 1980, Dodd and Jensen worked out inner models for strong cardinals. Building on the previous work of Mitchell, Dodd and Jensen formulated coherent sequences of extenders, built inner models relative to such, and established GCH in these models. The arguments were based on extending the established techniques of securing iterability and comparison through coiteration. The GCH result was significant as precursory for the further developments in inner model theory based on "iteration trees". Thus, with extenders the inner model theory was carried forward to encompass strong cardinals, newly arguing for the coherence and consistency of the concept. There would however be little further progress until 1985, for the aspiration to encompass stronger hypotheses had to overcome the problem of "overlapping extenders", having to carry out comparison through coiteration for local structures built on $\left(\kappa_{1}, \beta_{1}\right)$-extenders and $\left(\kappa_{2}, \beta_{2}\right)$-extenders with $\kappa_{1} \leq \kappa_{2}<\beta_{1}$. The difficulty here is one of "moving generators": if an extender ultrapower is taken with a $\left(\kappa_{1}, \beta_{1}\right)$-extender and then with a $\left(\kappa_{2}, \beta_{2}\right)$-extender, then $\kappa_{2}<\beta_{1}$ implies that the generating features of the first extender ultrapower has been shifted by the second ultrapower and so one can no longer keep track of that ultrapower in the coiteration process. In any event, a crucial inheritance from this earlier work was the Dodd-Jensen Lemma about the minimality of iterations copied across embeddings, which would become crucial for all further work in inner model theory.

Through the 1970s and into the 1980s, those in the burgeoning field of settheoretic topology continued the topological investigation of the continuum and 
general, transfinite spaces, and large cardinals figured more and more as strong propositions were studied. One prominent problem was whether every normal Moore space was metrizable. Notably, William Fleissner [1982] showed that were that the case there would be an inner model with a measurable cardinal. With large cardinals intimated, Peter Nyikos [1980] then established that a certain instrumental "axoim" implies that every normal Moore space is metrizable, an axiom which Kunen had shown holds if $\kappa$ random reals are adjoined to a strong compact cardinal $\kappa$. Hence, if there is a strong compact cardinal, then in a forcing extension every normal Moore space is metrizable. With this as one striking example, large cardinals, and even the Proper Forcing Axiom, began to play substantial roles in new relative consistency results in this area feature general topological articulations. The rich and wide-ranging Handbook of Set-Theoretic Topology [Kunen and Vaughn, 1984] summed up the progress, and its many articles set the tone for further work.

Conspicuous in combinatorics and topology would be the work of Stevo Todorcevic. Starting with his doctoral work with Kurepa, in 1979 he carried out an incisive analysis of uncountable trees - Suslin, Aronszajn, Kurepa trees and variants - and their linearizations and isomorphism types. In 1983 he [1984] dramatically reoriented the sense of strength for the Proper Forcing Axiom by showing that PFA implies that $\square_{\kappa}$ fails for every $\kappa>\omega$. PFA had previously been shown consistent relative to the existence of a supercompact cardinal. With the failure of $\square_{\kappa}$ for singular $\kappa$ having been seen as having quite substantial consistency strength, PFA was itself seen for the first time as a very strong proposition. Todorcevic would go from strength to strength, making substantial contributions to the theory of partition relations, eventually establishing definitive results about $\omega_{1}$ as the archetypal uncountable order-structure (cf. his book [2007]).

Starting in 1980 Foreman made penetrating inroads into the possibilities for very strong propositions holding low in the cumulative hierarchy based on the workings of generic elementary embeddings. Extending Kunen's work and deploying Silver's master condition idea, Foreman [1982] initially used 2-huge cardinals to get modeltheoretic transfer principles to hold and [1983] saturated ideals to exist among the range of $\aleph_{n}$ 's. He would soon focus on generic elementary embeddings and corresponding ideals themselves, even making them postulational for set theory.

In a major 1984 collaboration in Jerusalem, Foreman, Magidor, and Shelah (cf. [1984]) established penetrating results that led to a new understanding of strong propositions and the possibilities with forcing. The focus was on a new, maximal forcing axiom: A partial order $P$ preserves stationary subsets of $\omega_{1}$ iff stationary subsets of $\omega_{1}$ remain stationary in any forcing extension by $P$, and with this we have Martin's Maximum (MM): For any $P$ preserving stationary subsets of $\omega_{1}$ and collection $\mathcal{D}$ of $\aleph_{1}$ dense subsets of $P$, there is a filter $G \subseteq P$ meeting every member of $\mathcal{D}$. This subsumes PFA and is a maximally strong forcing axiom in that there is a $P$ which does not preserve stationary subsets of $\omega_{1}$ for which the conclusion fails. Foreman, Magidor, and Shelah established: If there is a supercompact cardinal $\kappa$, then there is a forcing extension in which $\kappa=\omega_{2}$ and 
MM holds.

Shelah had considered a weakening of properness called semiproperness, a notion for forcing that could well render uncountable cofinalities countable. To iterate such forcings, it had to be faced that the countable cofinality of limit stages cannot be ascertained in advance, and so he developed revised countable support iteration (RCS) based on names for the limit stage indexing. Foreman, Magidor, and Shelah actually carried out Baumgartner's PFA consistency proof for semiproper forcings with RCS iteration to establish the consistency of the analogous Semiproper Forcing Axiom (SPFA). Their main advance was that, although a partial order that preserves stationary subsets of $\omega_{1}$ is not necessarily semiproper, it is in this supercompact collapsing context. ${ }^{13}$

Foreman, Magidor, and Shelah then established the relative consistency of several propositions by deriving them directly from MM. One such proposition was that $\mathrm{NS}_{\omega_{1}}$ is $\aleph_{2}$-saturated. Hence, not only was the upper bound for the consistency strength of having an $\aleph_{1}$-complete $\aleph_{2}$-saturated ideal over $\omega_{1}$ considerably reduced from Kunen's huge cardinal, but for the first time the consistency of $\mathrm{NS}_{\omega_{1}}$ itself being $\aleph_{2}$-saturated was established relative to large cardinals. Another formative result was simply that MM actually implies that $2^{\aleph_{0}}=\aleph_{2}$, starting a train of thought about forcing axioms actually determining the size of the continuum. ${ }^{14}$

With their work Foreman, Magidor, and Shelah had overturned a long-held view about the scaling down of large cardinal properties. In the first flush of new hypotheses and propositions, Kunen had naturally enough collapsed a large cardinal to $\omega_{1}$ in order to transmute strong properties of the cardinal into an $\aleph_{1-}$ complete $\aleph_{2}$-saturated ideal over $\omega_{1}$, and this sort of direct connection had become the rule. The new discovery was that a collapse of a large cardinal to $\omega_{2}$ instead can provide enough structure to secure such an ideal. In fact, Foreman, Magidor, and Shelah showed that even the usual Levy collapse of a supercompact cardinal to $\omega_{2}$ engenders an $\aleph_{1}$-complete $\aleph_{2}$-saturated ideal over $\omega_{1}$. In terms of method, the central point is that the existence of sufficiently large cardinals implies the existence of substantial generic elementary embeddings with small critical points like $\omega_{1}$. Woodin's later strengthenings and elaborations of these results would have far-reaching consequences.

\subsection{Reflecting stationary sets}

Here we work in a discussion of stationary set reflection. This serves mainly as an illustrative example of a theme that interweaves through the mainstream of large cardinals, but could well be considered a topic sufficiently prominent to warrant separate description, particularly because of the way it displays aspects of many emerging issues. Another theme that would have served as well is Chang's

\footnotetext{
${ }^{13}$ Eventually, Shelah [1987] did establish that MM and SPFA are equivalent.

${ }^{14}$ It would be by different and elegant means that Todorcevic would show in 1990 (cf. [Bekkali, 1991]) that PFA already implies that $2^{\aleph_{0}}=\aleph_{2}$.
} 
Conjecture. ${ }^{15}$

For $S \subseteq \kappa$ stationary in $\kappa, S$ reflects iff there an $\alpha<\kappa$ such that $S \cap \alpha$ is stationary in $\alpha$. In these terms, we let $\operatorname{Refl}(S)$ be the assertion that every stationary subset of $S$ reflects. That $S \subseteq \kappa$ is stationary in $\kappa$ is a basic "secondorder" property of $\langle\kappa, S\rangle$, and with reflection having become an important heuristic in set theory, stationary set reflection commended itself as a specific, second-order reflection possibility for investigation. It is a direct consequence of a characterizing reflection property of weakly compact cardinals that if $\kappa$ is weakly compact, then $\operatorname{Refl}(\kappa)$. Jensen [1972] raised interest in stationary set reflection by getting the converse in $L$ as an initial dividend of his fine structure theory: If $V=L$, then $\kappa$ is weakly compact iff $\operatorname{Refl}(\kappa)$. On the other hand, Kunen in 1972 (cf. [1978]) quickly complemented Jensen's result by showing: If $\kappa$ is weakly compact, then there a cardinal-preserving forcing extension in which $\kappa$ is inaccessible but not weakly compact, yet $\operatorname{Refl}(\kappa)$ holds. Kunen introduced a way of forcing a $\kappa$-Suslin tree through a large cardinal $\kappa$, thereby precluding its weak compactness, in such a way that the forcing combined by the further forcing for shooting a cofinal branch through the adjoined tree is essentially the same as having just added one Cohen subset of $\kappa$ in the first place. For the actual model, Kunen made an initial application of Silver's "reversed Easton" technique (cf. 3.3) by adjoining for every inaccessible $\lambda<\kappa$ a Cohen subset of $\lambda$, and then followed this with his way of adjoining a $\kappa$-Suslin tree. Shooting a cofinal branch through the tree would then resurrect the large cardinal properties of $\kappa$ because of the reversed Easton preparation, and Kunen exploited this promixity to show that $\operatorname{Refl}(\kappa)$ already holds in his model.

What about $\operatorname{Refl}(\kappa)$ for successor cardinals $\kappa$ ? For regular $\lambda<\kappa$, define the paradigmatic stationary sets

$$
S_{\lambda}^{\kappa}=\{\alpha<\kappa \mid \operatorname{cf}(\alpha)=\lambda\} .
$$

Then for regular $\lambda$, clearly $S_{\lambda}^{\lambda^{+}}$cannot reflect. Beyond this initial restriction, forcing and $L$ expectedly work against stationary set reflection: For any regular $\kappa \geq \omega_{2}$, a non-reflecting stationary subset of $S_{\omega}^{\kappa}$ can be adjoined by straightforward forcing with initial segments. The Jensen combinatorial principle $\square_{\lambda}$, holding in $L$, directly implies that $\operatorname{Refl}(S)$ fails for every stationary $S \subseteq \lambda^{+}$.

Large cardinals provided the counterweight. Reminiscent of the Mitchell-Silver result on the tree property (cf. 3.3) but more immediate in its proof, Baumgartner [1976] observed how the collapse of a weakly compact cardinal preserves a significant attribute: If a weakly compact cardinal is Levy collapsed to make it $\omega_{2}$, then $\operatorname{Refl}\left(S_{\omega}^{\omega_{2}}\right)$ holds in the extension. $\operatorname{Refl}\left(S_{\omega}^{\omega_{2}}\right)$ implies the failure of $\square_{\omega_{1}}$, which by Jensen's fine structure argument for the principle holding in $L$ implies that $\omega_{2}$ must be Mahlo there. Thus, as for many propositions in modern set theory,

\footnotetext{
${ }^{15}$ For some representative articles, [Silver, 1971b] established the consistency of Chang's Conjecture relative to a large cardinal; Donder (cf. [Donder et al., 1981]) established the converse with the core model $K^{\mathrm{DJ}}$; [Donder and Koepke, 1983] investigated higher versions; and [Levinski et al., 1990] established the relative consistency of the version at $\aleph_{\omega+1}$.
} 
the consistency strength of $\operatorname{Refl}\left(S_{\omega}^{\omega_{2}}\right)$ was initially bracketed, here between having a weakly compact cardinal and having a Mahlo cardinal. The expectation was nonetheless for weak compactness. This was especially so as Magidor (cf. [1982]) showed that if for any pair of sets $S_{1}, S_{2} \subseteq \omega_{2}$ both stationary in $\omega_{2}$ there is an $\alpha<\omega_{2}$ such that both $S_{1} \cap \alpha$ and $S_{2} \cap \alpha$ are stationary in $\alpha$, then $\omega_{2}$ is weakly compact in $L$. This "pair reflection" actually held in the Levy collapse of a weakly compact cardinal to make it $\omega_{2}$ by checking Baumgartner's argument, and so at least there was this equi-consistency.

It was somewhat surprising then, when in the late 1970s, Leo Harrington and Shelah (cf. [1985]) established: If a Mahlo cardinal, after first arranging $\mathrm{CH}$, is Levy collapsed to make it $\omega_{2}$, then $\operatorname{Refl}\left(S_{\omega}^{\omega_{2}}\right)$ holds in the extension. Their result involved a refined auxiliary forcing argument, which revealed a sense in which a concrete second-order reflection requires only a first-order overlay as given by a Mahlo cardinal. Remarkably, reflection and pair reflection were exactly and separately gauged in consistency strength!

Shelah also noted a generalization of Baumgartner's result, one exhibiting the global reflection possibilities provided by supercompactness: If a supercompact cardinal is Levy collapsed to make it $\omega_{2}$, then in the extension $\operatorname{Refl}\left(S_{\omega}^{\kappa}\right)$ holds for every regular $\kappa \geq \omega_{2}$.

As in many situations, $\omega_{2}$ typified an accessible cardinal in the investigation of the consistency strength of stationary set reflection, as described above, but with $\omega_{2}$ paradigmatic of successors of regular cardinals. For successors of singular cardinals the emerging inner model theory showed that the failure of $\square_{\kappa}$ for singular $\kappa$ has strong consistency strength, and so also $\operatorname{Refl}\left(\kappa^{+}\right)$. With $\omega_{\omega+1}$ typifying a successor of a singular cardinal, Magidor [1982] established: If infinitely many supercompact cardinals are iteratively Levy collapsed to become the $\omega_{n}$ 's, then in the extension $\operatorname{Refl}\left(\omega_{\omega+1}\right)$ holds. The intricate proof involved the lifting of various supercompact embeddings to the extension and then a careful analysis of how stationary reflection via these embeddings at intermediate stages is preserved to the end. This result, like others of Magidor's from this period, featured the liberal use of strong hypotheses providing strong elementary embeddings. In this case however, the hypothesis of the theorem has not as yet been significantly reduced in subsequent work for getting $\operatorname{Refl}\left(\omega_{\omega+1}\right)$. As with several strong propositions, it has been sufficient that a beachhead was secured, and a stalking for equi-consistency has not proceeded - unlike for the Singular Cardinals Problem (cf. 4.4) with its immediacy as a pressing issue for set theory. Be that as it may, Magidor's result was a seminal one for the investigation of the combinatorics and consistency of properties at successors of singular cardinals, a subject which has intimately involved strong large cardinal hypotheses through forcing. ${ }^{16}$

The 1984 Foreman-Magidor-Shelah collaboration led to the formulation of a strong stationary set reflection principle. The sets $\mathcal{P}_{\kappa} X=\{x \subseteq X|| x \mid<\kappa\}$ having emerged in the ultrafilter formulations of large large cardinals (cf. 3.1), the concepts of closed unbounded and stationary have natural generalizations to

${ }^{16}$ See Eisworth's chapter in [Foreman and Kanamori, 2010]. 
this index set: $C \subseteq \mathcal{P}_{\kappa} X$ is closed unbounded if it is closed, i.e. under the taking of $\subseteq$-chains of length less than $\kappa$, and unbounded, i.e. for any $y \in \mathcal{P}_{\kappa} X$ there is an $x \supseteq y$ such that $x \in C . S \subseteq \mathcal{P}_{\kappa} X$ is stationary if $C \cap S \neq \emptyset$ for any closed unbounded $C \subseteq \mathcal{P}_{\kappa} X$. Foreman, Magidor, and Shelah showed that their Martin's Maximum implies the following Reflection Principle: For any regular $\lambda \geq \omega_{2}$, if $S \subseteq \mathcal{P}_{\omega_{1}} \lambda$ is stationary, then there is an $X \subseteq \lambda$ with $|X|=\aleph_{1}$ and $X \supseteq \omega_{1}$ such that $S \cap \mathcal{P}_{\omega_{1}} X$ is stationary in $\mathcal{P}_{\omega_{1}} X$. Remarkably, the main-cited consequences of MM already follow from this reflection principle, except that it only implies that $\mathrm{NS}_{\omega_{1}}$ is precipitous. Todorcevic (cf. [Bekkali, 1991]) formulated a strong reflection principle, one that also follows from MM but moreover has as a consequence that $\mathrm{NS}_{\omega_{1}}$ is $\aleph_{2}$-saturated. Qi Feng and Jech [1998] also came up with a stronger reflection principle, but they soon showed it to be equivalent to Todorcevic's. Thus, stationary set reflection itself garnered a historically focal goal of large cardinal hypotheses.

\subsection{Consistency of determinacy}

The developments of the 1980s which are the most far-reaching and presentable as sustained narrative have to do with the stalking of the consistency of determinacy. By the late 1970s a more or less complete structure theory for the projective sets was in place, a resilient edifice founded on determinacy with both strong buttresses and fine details. In 1976 the researchers had started the Cabal Seminar in the Los Angeles area, and in a few years, with John Steel and Woodin having joined the ranks, attention began to shift to sets of reals beyond the projective sets, to inner models, and to questions of overall consistency. Most of the work before the crowning achievements of the later 1980s appears in the several proceedings of the Cabal Seminar, [Kechris and Moschovakis, 1978; Kechris et al., 1981; Kechris et al., 1983; Kechris et al., 1988].

The question of the overall consistency of determinacy came increasingly to the fore. Is $\mathrm{AD}$ consistent relative to some large cardinal hypothesis? Or, with its strong consequences, can AD subsume large cardinals in some substantial way or be somehow orthogonal? A decade after his initial result that the existence of a measurable cardinal implies $\boldsymbol{\Pi}_{1}^{1}$-determinacy, Martin [1980] applied its homogeneity idea with a "Martin-Solovay" tree representation for $\boldsymbol{\Pi}_{2}^{1}$ sets, together with algebraic properties of elementary embeddings posited close to Kunen's large cardinal inconsistency, to establish $\Pi_{2}^{1}$-determinacy. A direction was set but generality only came in 1984, when Woodin showed that an even stronger large cardinal hypothesis implies $\mathrm{AD}^{L(\mathbb{R})}$. So, a mooring was secured for $\mathrm{AD}$ after all in the large cardinal hierarchy. With Woodin's hypothesis apparently too remote it would now be a question of scaling it down according to the methods becoming available for proofs of determinacy, perhaps even achieving an equi-consistency result.

The rich 1984 Foreman-Magidor-Shelah work would have crucial consequences for the stalking of consistency also for determinacy. Shelah carried out a version of their collapsing argument that does not add any new reals but nonetheless 
gets an $\aleph_{1}$-complete $\aleph_{2}$-saturated ideal over $\omega_{1}$. Woodin then pointed out that with no new reals adjoined the generic elementary embedding induced by such an ideal can be used to establish that the ground model $L(\mathbb{R})$ reals are actually Lebesgue measurable. Thus Shelah and Woodin (cf. their [1990]) had established an outright result: If there is a supercompact cardinal, then every set of reals in $L(\mathbb{R})$ is Lebesgue measurable. This result not only portended the possibility of getting $\mathrm{AD}^{L(\mathbb{R})}$ from a supercompact cardinal, but through the specifics of the argument stimulated the reducing of the hypothesis. While Woodin was visiting Jerusalem in June 1984, he came up with what is now known as a Woodin cardinal. The hypothesis was then reduced as follows: If there are infinitely many Woodin cardinals with a measurable cardinal above them, then every set of reals in $L(\mathbb{R})$ is Lebesgue measurable. An early suggestion of optimality of hypothesis was that if the "infinitely" is replaced by " $n$ " for some $n \in \omega$, then one can conclude that every $\boldsymbol{\Sigma}_{n+2}^{1}$ set of reals is Lebesgue measurable. The measurable cardinal hovering above would be a recurring theme, the purpose loosely speaking to maintain a stable environment with the existence of sharps.

Especially because of its subsequent centrality, it is incumbent to give an operative definition of Woodin cardinal: For a set $A, \kappa$ is $\alpha$-A-strong iff there is an elementary embedding $j: V \rightarrow M$ witnessing that $\kappa$ is $\alpha$-strong which moreover preserves $A$ : $A \cap V_{\alpha}=j(A) \cap V_{\alpha}$. A cardinal $\delta$ is Woodin iff for any $A \subseteq V_{\delta}$, there is a $\kappa<\delta$ which is $\alpha$-A-strong for every $\alpha<\delta$.

A Woodin cardinal, evidently a technical, consistency-wise strengthening of a strong cardinal, is an important example of concept formation through method. The initial air of contrivance gives way to seeing that Woodin cardinal seemed to encapsulate just wanted is needed to carry out the argument for Lebesgue measurability. That argument having been based on first collapsing a large cardinal to get a saturated ideal and then applying the corresponding generic elementary embedding, Woodin later in 1984 stalked the essence of method and formulated stationary tower forcing (cf. [1990]). An outgrowth of the Foreman-Magidor-Shelah work, this notion of forcing streamlines their forcing arguments to show that a Woodin cardinal suffices to get a generic elementary embedding $j: V \rightarrow M$ with critical point $\omega_{1}$ and ${ }^{\omega} M \subseteq M$. With a new, minimizing large cardinal concept isolated, there would now be dramatic new developments both in determinacy and inner model theory. One important scaling down result was the early 1985 result of Shelah (cf. [1987a]): If $\kappa$ is Woodin, then in a forcing extension $\kappa=\omega_{1}$ and $\mathrm{NS}_{\omega_{1}}$ is $\aleph_{2}$-saturated. The large cardinal strength now seemed minimal for getting such an ideal, and there was anticipation of achieving an equi-consistency.

Steel in notes of Spring 1985 developed an inner model for a weak version of Woodin cardinal. While inner models for strong cardinals had only required linear iterations for comparison, the new possibility of overlapping extenders and moving generators had led Mitchell in 1979 to develop iteration trees of iterated ultrapowers for searching for possible well-founded limits of models along branches. A particularly simple example of an iteration tree is an alternating chain, a tree consisting of two $\omega$-length branches with each model in the tree an extender ultrapower 
of the one preceding it on its branch, via an extender taken from a corresponding model in the other branch. Initially, Steel tried to avoid alternating chains, but the Foreman-Magidor-Shelah work showed that for dealing with Woodin cardinals they would be a necessary part. Their use soon led to a major breakthrough in the investigation of determinacy.

In the Fall of 1985 Martin and Steel (cf. [1988; 1989]) showed that Woodin cardinals imply the existence of alternating chains in which both branches have well-founded direct limits, and used this to establish: If there are infinitely many Woodin cardinals, then PD holds. This was a culmination of method in several respects. In the earlier Martin results getting $\boldsymbol{\Pi}_{1}^{1}$-Determinacy and $\boldsymbol{\Pi}_{2}^{1}$-Determinacy, trees on $\omega \times \kappa$ for some cardinal $\kappa$ had been used, to each node of which were attached ultrafilters in a coherent way that governed extensions. Kechris and Martin isolated the relevant concept of homogeneous tree, the point being that sets of reals which are the projections $p[T]$ of such trees $T$ - the homogeneously Suslin sets are determined. With PD, the scale property had been propagated through the projective hierarchy. Now with Woodin cardinals, having representations via homogeneous trees was propagated, getting determinacy itself. In particular, Martin and Steel established: If $n \in \omega$ and there are $n$ Woodin cardinals with a measurable cardinal above them, then $\boldsymbol{\Pi}_{n+1}^{1}$-determinacy holds.

Within weeks after the Martin-Steel breakthrough, Woodin used it together with stationary towers to investigate tree representations in $L(\mathbb{R})$ to establish: If there are infinitely Woodin cardinals with a measurable cardinal above them, then $\mathrm{AD}^{L(\mathbb{R})}$ holds. With the consistency strength of $\mathrm{AD}$ having been gauged by this result, Woodin soon established the crowning equi-consistency result: The Axiom of Determinacy is equi-consistent with the existence of infinitely many Woodin cardinals. $^{17}$

This was a remarkable achievement of the concerted effort to establish the consistency strength of AD along the large cardinal hierarchy. But even this would just be a beginning for Woodin, who would go from strength to strength in establish many structural results involving AD and stronger principles, to become preeminent with Shelah in set theory.

\subsection{Into the $1990 \mathrm{~s}$}

In the later 1980s set theory continued to expand apace in various directions, and we conclude our historical survey by mentioning here a few of the most prominent developments, each of a different character but all being decisive advances.

In inner model theory, Stewart Baldwin in 1987 made a suggestion, one which Mitchell then newly forwarded, which led to a crucial methodological advance. Up to then, the extender models $L[\vec{E}]$ constructed relative to a coherent sequence of extenders $\vec{E}$ had each extender $E$ in the sequence "measure" all the subsets in $L[\vec{E}]$ of the critical point. The Baldwin-Mitchell idea was to construct only with

\footnotetext{
${ }^{17}$ See the Neeman and Koellner-Woodin chapters of [Foreman and Kanamori, 2010] for proofs of each direction benefitting from hindsight.
} 
"partial" extenders $E$ which if indexed at $\gamma$ only measures the sets in $L_{\gamma}[\vec{E}\lceil\gamma]$. This together with a previous Mitchell strategy of carrying out the comparison process using finely calibrated partial ultrapowers ("dropping to a mouse") led to a comparison process internal to $L[\vec{E}]$ based on the use of fine structure. The infusion of fine structure made the development of the new extender models more complex, but with this came the important dividends of a more uniform presentation, a much stronger condensation, and a more systematic comparison process. During 19879, Mitchell and Steel (cf. their [1994]) worked out the details and showed that if there is a Woodin cardinal then there is an inner model $L[\vec{E}], L$-like in satisfying $\mathrm{GCH}$ and so forth, in which there is a Woodin cardinal. The process involved the correlating of iteration trees for $L[\vec{E}]$ with iteration trees in $V$. A canonical, fine structural inner model of a Woodin cardinal newly argued for the consistency of the concept, as well as provided a great deal of understanding about it as set in a finely tuned, layer-by-layer hierarchy.

What about a core model "up to" a Woodin cardinal, in analogy to $K^{\mathrm{DJ}}$ for $L[U]$ ? In 1990, Steel (cf. [1996]) solved the "core model iterability problem" by showing that large cardinals in $V$ are not necessary for showing that certain models $L[\vec{E}]$ have sufficient iterability properties. With this, he constructed a new core model, first building a "background certified" $K^{c}$ based on extenders in $V$ and then the "true" core model $K$. Steel was thus able to extend the previous work of Mitchell on the core model $K[\mathcal{U}]$ up to $\exists \kappa\left(o(\kappa)=\kappa^{++}\right)$to establish e.g.: If there is an $\aleph_{1}$-complete $\aleph_{2}$-saturated ideal over $\omega_{1}$ and a measurable cardinal, then there is an inner model with a Woodin cardinal. Thus, Shelah's 1985 forcing result and Steel's, except for the artifact of "the measurable cardinal above", had calibrated an important consistency strength, and what had become a central goal of forcing and inner model theory was handily achieved.

In the early 1990s, Steel, Mitchell, and Ernest Schimmerling (cf. their [1997]) pushed the Jensen covering argument over the hurdles of the new fine structural Steel core model $K$ to establish a covering lemma up to a Woodin cardinal. Schimmerling [1994] both established combinatorial principles in $K$ as well established new consistency strengths, e.g. PFA implies that there is an inner model with a Woodin cardinal.

The later 1980s featured a distinctive development that led to a new conceptual framework of applicability to singular cardinals, new incisive results in cardinal arithmetic, and a re-orienting of set theory to new possibilities for outright theorems of ZFC. Starting in late 1987 Shelah returned to the work on bounds for powers of singular cardinals and drew out an extensive underlying structure of possible cofinalities of reduced products, soon codified as pcf theory. With this emerged new work in singular cardinal combinatorics, with Shelah himself initially providing applications to model theory, partition relations, Jónsson algebras, Boolean algebras, and cardinal arithmetic. This last was epitomized by a dramatic result that exhibited how the newly seen structural constraints impose a tight bound: If $\delta$ is a limit ordinal with $|\delta|^{\mathrm{cf}(\delta)}<\aleph_{\delta}$ then $\aleph_{\delta}^{\mathrm{cf}(\delta)}<\aleph_{\left(|\delta|^{+4}\right)}$, so that in particular if $\aleph_{\omega}$ is a strong limit cardinal, then $2^{\aleph_{\omega}}<\aleph_{\omega_{4}}$. This substantively 
betters the former bound $\aleph_{\left(2^{\aleph_{0}}\right)}$ of his 1980 result (cf. [1982]), and complements his [1983] consistency result from that time that values for $2^{\aleph_{\omega}}$ up to $\aleph_{\omega_{1}}$ are possible. Whether the gap betweeen $\aleph_{\omega_{1}}$ and $\aleph_{\omega_{4}}$ can be closed is a prominent open problem.

Suppose that $A$ is an infinite set of cardinals and $F$ is a filter over $A$. The product $\Pi A$ consists of functions $f$ with domain $A$ such that $f(a) \in a$ for every $a \in A$. For $f, g \in \Pi A$, the relation $=_{F}$ defined by $f={ }_{F} g$ iff $\{a \in A \mid f(a)=g(a)\} \in F$ is an equivalence relation on $\Pi A$, and the reduced product $\Pi A / F$ consists of the equivalence classes. We can impose order, officially on $\Pi A / F$ but still working with functions themselves, by: $f<_{F} g$ iff $\{a \in A \mid f(a)<g(a)\} \in F$.

Shelah's new theory took as central the investigation of the possible cofinalities function:

$$
\operatorname{pcf}(A)=\{\operatorname{cf}(\Pi A / D) \mid D \text { is an ultrafilter over } A\}
$$

as calibrated by the ideals

$$
\begin{aligned}
& J_{<\lambda}[A]=\{b \subseteq A \mid \operatorname{cf}(\Pi A / D)<\lambda \text { whenever } \\
& D \text { is an ultrafilter over } A \text { such that } b \in D\} .
\end{aligned}
$$

These concepts had appeared before in Shelah's work, notably in his 1980 result $\aleph_{\delta}^{\mathrm{cf}(\delta)}<\aleph_{\left(|\delta|^{\mathrm{cf}(\delta)}\right)^{+}}$, but now they became autonomous and were propelled forward by the discovery of unexpectedly rich structure.

With an eye to substantive cofinal subsets $A$ of a singular cardinal, the abiding assumption was that $A$ is a set of regular cardinals satisfying $|A|<\min (A)$. With this one gets that for any ultrafilter $D$ over $A, \operatorname{cf}(\Pi A / D)<\lambda$ iff $D \cap J_{<\lambda}[A] \neq \emptyset$, and further, that $\operatorname{pcf}(A)$ has a maximum element. At the heart is the striking result that $J_{<\lambda^{+}}[A]$ is generated by $J_{<\lambda}[A]$ together with a single set $B_{\lambda} \subseteq A$. Shelah in fact got "nice" generators $B_{\lambda}$ derived from imposing the structure of elementary substructures of a sufficiently large $H(\Psi)$. A bulwark of the theory is then that with $\mu=\sup A$ and $F=\{X \subseteq \operatorname{cf}(\mu)|| \operatorname{cf}(\mu)-X \mid<\operatorname{cf}(\mu)\}$ the filter of co-bounded subsets of $\operatorname{cf} \mu$, it is a theorem of ZFC that $\Pi A / F$ has a linearly ordered, cofinal sequence of length $\mu^{+}$-a scale for $\mu$. The careful control on the possible cofinalities led as well, when $A$ consists of all the regular cardinals in an interval of cardinals, to $|\operatorname{pcf}(A)| \leq|A|^{+++}$, and in particular to the $\aleph_{\omega_{4}}$ bound mentioned above.

Shelah's work on pcf theory to 1993 appeared in his book Cardinal Arithmetic [1994], and since then he has further developed the theory and provided wideranging applications. Through its applicability pcf theory has to a significant extent been woven into modern set theory as part of the ZFC facts of singular cardinal combinatorics.

The Singular Cardinal Hypothesis (SCH) and the train of results starting with the Prikry-Silver result of the early 1970s were to be decisively informed by results of Moti Gitik. Gitik's work exhibits a steady engagement with central and difficult issues of set theory and a masterful virtuosity in the application of sophisticated techniques over a broad range. Gitik [1980] had established through an iterated 
Prikry forcing the conspicuous singularization result that: If there is a proper class of strongly compact cardinals, then in a ZF inner model of a class forcing extension every infinite cardinal has cofinality $\omega$. Mentioned earlier was the mid-1970s result that that $\mathrm{NS}_{\omega_{1}}$ is precipitous is equi-consistent with having a measurable cardinal. Gitik [1984] established: The precipitousness of $\mathrm{NS}_{\omega_{2}}$ is equi-consistent with having a measurable cardinal $\kappa$ such that $o(\kappa)=2$ in the Mitchell order. The difficult, forcing direction required considerable ingenuity because of inherent technical obstructions. ${ }^{18}$

Turning to the work on SCH, in 1988 Woodin dramatically weakened the large cardinal hypothesis needed to get a measurable cardinal $\kappa$ satisfying $2^{\kappa}>\kappa^{+}$, using a technique of "altering generic objects". With the subsequent use of Prikry forcing leading to the failure of $\mathrm{SCH}$, a proposition technically strengthening measurability now sufficed for the failure of SCH. He also showed that one can in fact get Magidor's conclusion that $\aleph_{\omega}$ could be the least cardinal at which GCH fails. Soon afterwards Gitik established both directions of an equi-consistency: First, he established that one can get the consistency of Woodin's proposition from just $\exists \kappa\left(o(\kappa)=\kappa^{++}\right)$. Then, he applied a result from Shelah's pcf theory to Mitchell's $K[\mathcal{U}]$ analysis to establish, bettering a previous result of Mitchell, that $\exists \kappa\left(o(\kappa)=\kappa^{++}\right)$is actually necessary to get the failure of SCH. Hence, The failure of $\mathrm{SCH}$ is equi-consistent with $\exists \kappa\left(o(\kappa)=\kappa^{++}\right)$.

Woodin's model in which GCH first fails at $\aleph_{\omega}$ required a delicate construction to arrange GCH below and an ingenious idea to get $2^{\aleph_{\omega}}=\aleph_{\omega+2}$. How about getting $2^{\aleph_{\omega}}>\aleph_{\omega+2}$ ? In a signal advance of method, Gitik and Magidor in 1989 provided a new technique to handle the general Singular Cardinals Problem with appropriately optimal hypotheses. The Prikry-Silver two-stage approach, first making $2^{\kappa}$ large and then singularizing $\kappa$ without adding any new bounded subsets or collapsing cardinals, had been the basic model for attacking the Singular Cardinals Problem. Gitik and Magidor showed how to add many subsets to a large cardinal $\kappa$ while simultaneously singularizing it without adding any new bounded subsets or collapsing cardinals. Thus, it became much easier to arrange any particular continuum function behavior below $\kappa$, like achieving $\mathrm{GCH}$ below, while at the same time making $2^{\kappa}$ large. Moreover, the new method smacked of naturalness and optimality.

The new Gitik-Magidor idea was to add many new Prikry $\omega$-sequences corresponding to $\kappa$-complete ultrafilters over $\kappa$ while maintaining the basic properties of Prikry forcing. There is an evident danger that if these Prikry sequences are too independent, information can be read from them that corresponds to new reals being adjoined. The solution was to start from a sufficient strong large cardinal hypothesis and develop an extender-based Prikry forcing structured on a "nice system" of ultrafilters $\left\langle U_{\alpha} \mid \alpha<\lambda\right\rangle$, a system such that for many $\alpha \leq \beta<\lambda$ there is a ground model function $f: \kappa \rightarrow \kappa$ such that: For all $X \subseteq \kappa, X \in U_{\alpha}$ iff $f^{-1}(X) \in U_{\beta}$. (Having such a projection function is the classical way of connecting two ultra-

\footnotetext{
${ }^{18}$ Gitik eventually established $[1995 ; 1997]$ definitive equi-consistency results for having $\mathrm{NS}_{\kappa}$ precipitous for $\kappa>\omega^{2}$ of analogous sort involving the Mitchell order.
} 
filters together, and one writes that $U_{\alpha} \leq_{\mathrm{RK}} U_{\beta}$ under the Rudin-Keisler partial order.) By this means one has the possibility of adding new subsets of $\kappa$, corresponding to different Prikry sequences, which are still dependent on each other so that no new bounded subsets need necessarily be added in the process. Gitik and Magidor worked out how their new approach leads to what turns out to be optimal or near optimal consistency results, and incorporating collapsing maps as in previous arguments of Magidor and Shelah, they got models in which GCH holds below $\aleph_{\omega}$ yet $2^{\aleph_{\omega}}=\aleph_{\alpha+1}$ for any prescribed countable ordinal $\alpha$.

In subsequent work Gitik, together with Magidor, Mitchell, and others, have considerably advanced the investigation of powers of singular cardinals. Equiconsistency results have been achieved for large powers of singular cardinals along the Mitchell order and with $\alpha$-strong cardinals, and uncountable cofinalities have been encompassed, the investigation ongoing and with dramatic successes.

\subsection{Ideals}

In this last section we take a quick look at later developments involving ideals. In the 1990s ideals low in the cumulative hierarchy yet having strong consistency strength became more and more focal as being of intrinsic interest, to the extent that postulations about them were put forward as themselves large cardinal axioms as modulated by forcing.

The continuing investigation of determinacy led to the study of a very strong property of ideals. An ideal $I$ over a cardinal $\kappa$ is $\lambda$-dense iff there is a family $D \subseteq P(\kappa)-I$ with $|D|=\lambda$ such that for any $X \in P(\kappa)$, there is a $Y \in D$ such that $Y-X \in I$. This is a natural notion of density for the algebra $P(\kappa)-I$. Clearly, if $I$ is $\lambda$-dense and $\lambda<\mu$, then $I$ is $\mu$-saturated. Woodin drew attention to $\aleph_{1}$ complete $\aleph_{1}$-dense ideals over $\omega_{1}$ by establishing the consistency of their existence first from strong determinacy hypotheses and, by a notably simple argument, from a large cardinal hypothesis just weaker than having a huge cardinal. Stimulating interest on how strong ideals over $\omega_{1}$ may affect $\mathrm{CH}$, Shelah [1986] showed by an elegant argument that ZFC imposes an intriguing limitation: If $2^{\aleph_{0}}<2^{\aleph_{1}}$ (e.g. if $\mathrm{CH}$ holds), then $\mathrm{NS}_{\omega_{1}}$ is not $\aleph_{1}$-dense.

Pushing the connections between ideals and the consistency of determinacy, Woodin established the following, using results of Steel about $L(\mathbb{R})$ : If there is an $\aleph_{1}$-complete, $\aleph_{1}$-dense ideal over $\omega_{1}$, then $\mathrm{AD}^{L(\mathbb{R})}$ holds. Then in late 1992, Woodin was able to establish the following converse for consistency strength: $A s$ sume $\mathrm{AD}^{L(\mathbb{R})}$; then there is a forcing extension of $L(\mathbb{R})$ satisfying $\mathrm{ZFC}+\mathrm{NS}_{\omega_{1}}$ is $\aleph_{1}$-dense. Quite remarkably, $\mathrm{AD}$ is equi-consistent with $\mathrm{NS}_{\omega_{1}}$ being $\aleph_{1}$-dense! This encapsulation made ideal hypotheses central to modern set theory.

Is $\mathrm{NS}_{\omega_{1}}$ being $\aleph_{2}$-saturated consistent with $\mathrm{CH}$ ? This was a prominent question for some time, with the consistency results for $\aleph_{2}$-saturation all achieved with $\neg \mathrm{CH}$ and Shelah's actually deriving $\neg \mathrm{CH}$ from $\aleph_{1}$-denseness. Again it was Woodin; he established in descriptive set-theoretic terms: If $\mathrm{NS}_{\omega_{1}}$ is $\aleph_{2}$-saturated and there is a measurable cardinal, then $\boldsymbol{\delta}_{2}^{1}=\omega_{2}$. Since then there are, hierarchi- 
cally, prewellorderings of length at least $\omega_{2}$, $\mathrm{CH}$ fails in a strong, definable sense. Pursuing this work, Woodin in the mid-1990s (cf. [1999]) developed a vast machinery for forcing over models of determinacy, his $\mathbb{P}_{\max }$ extensions, that get at canonical maximal models of ZFC $+\neg \mathrm{CH}$. This in turn led (cf. [2001a; 2001b]) to his $\Omega$-logic, and a possible, structured solution to the Continuum Problem itself. With this we have peered ahead a long distance in a vast, remarkably rich direction; we return to the bedrock of large cardinals and forcing to draw this chapter to a close.

Foreman (cf. his chapter in [Foreman and Kanamori, 2010]) has provided a systematic approach to ideals and generic elementary embeddings, to the point of postulating axioms as was first done on his [1986]. To set things out once again, what is at play is the basic synthesis of forcing and ultrapowers whereby one starts with an ideal $I$ over a cardinal $\kappa$; forces with $P(\kappa)-I$ where $p$ is stronger than $q$ if $p-q \in I$; thus produces an ultrafilter over the ground model $P(\kappa)$; and then gets a "generic" elementary embedding of the ground model into the corresponding ultrapower. With the possibilities of ideals occurring low in the cumulative hierarchy, so that large cardinal ideas can be brought to bear on classical problems of set theory, an enormous subject has grown.

At the broadest level are the "three parameters" describing the strength of a generic elementary embedding $j: V \rightarrow M$ : how $j$ moves the ordinals; how large and closed $M$ is; and the nature of the forcing that provided $j$. This last is the new parameter at play beyond the "conventional" large cardinal hypotheses. Ideals through their forcing properties thus assuming a crucial role, another guiding theme is the distinction between "natural" ideals that have intrinsic definitions and ideals "induced" by elementary embeddings. Strong ideal assumptions gain an autonomy as "generic large cardinals" in their own right, and there is a further delineation according to consequences of generic large cardinals and consistency results about them.

Of consequences, we mentioned Shelah's and Woodin's $\neg \mathrm{CH}$ results above, and there are implications for $\mathrm{GCH}$; SCH; graphs and groups; stationary set reflection; Suslin and Kurepa trees; partition properties; and descriptive set theory. Moreover, there are remarkable limitative results on ideals properties e.g. the GitikShelah result (cf. their [1997]) that if $\kappa$ is regular and $\delta^{+}<\kappa$, then the ideal generated by $\mathrm{NS}_{\kappa}$ and $\{\alpha<\kappa \mid \operatorname{cf}(\alpha)=\delta\}$ is not $\kappa^{+}$-saturated, and their result (cf. their [1989]) that there is no $\aleph_{1}$-complete $\aleph_{0}$-dense nowhere prime ideal. ${ }^{19}$

Of consistency results, beyond the several we have already mentioned there is Foreman's relative consistency of having an $\aleph_{1}$-complete $\aleph_{1}$-dense (uniform) ideal over $\omega_{2}$ from a strong hypothesis about two coordinated huge-type cardinals. In general terms, Foreman has shown how the "conventional" large cardinals and the "generic" large cardinals interleave in consistency strength, sometimes with equi-consistencies at play. At this general confluence of the methods of forcing and ultrapowers, there is a wealth of possibilities.

\footnotetext{
${ }^{19} \mathrm{An}$ ideal is nowhere prime if it restricted to no set becomes a maximal ideal, dual to an
} ultrafilter 


\section{BIBLIOGRAPHY}

[Baumgartner, 1976] J. E. Baumgartner. A new class of order types. Annals of Mathematical Logic, 9:187-222, 1976.

[Baumgartner, 1983] J. E. Baumgartner. Iterated forcing. In A. R. D. Mathias, editor, Surveys in Set Theory, volume 87 of London Mathematical Society Lecture Note Series, pages 1-59. Cambridge University Press, 1983.

[Bekkali, 1991] M. Bekkali. Topics in Set Theory, volume 1476 of Lecture Notes in Mathematics. Springer-Verlag, 1991.

[Blackwell, 1967] D. Blackwell. Infinite games and analytic sets. Proceedings of the National Academy of Sciences USA, 58:1836-1837, 1967.

[Dales and Woodin, 1987] H. G. Dales and W. H. Woodin. An Introduction to Independence for Analysts, volume 115 of London Mathematical Society Lecture Note Series. Cambridge University Press, 1987.

[Devlin and Jensen, 1975] K. J. Devlin and R. B. Jensen. Marginalia to a theorem of Silver. In G. H. Müller, A. Oberschelp, and K. Potthoff, editors, ISILC Logic Conference, Kiel, 1974, volume 499 of Lecture Notes in Mathematics, pages 115-142. Springer, 1975.

[Devlin and Johnsbraten, 1974] K. J. Devlin and H. Johnsbraten. The Souslin Problem, volume 405 of Lecture Notes in Mathematics. Springer-Verlag, 1974.

[Dodd and Jensen, 1981] A. J. Dodd and R. B. Jensen. The core model. Annals of Mathematical Logic, 20:43-75, 1981.

[Dodd and Jensen, 1982a] A. J. Dodd and R. B. Jensen. The covering lemma for K. Annals of Mathematical Logic, 22:1-30, 1982.

[Dodd and Jensen, 1982b] A. J. Dodd and R. B. Jensen. The covering lemma for L[U]. Annals of Mathematical Logic, 22:127-135, 1982.

[Dodd, 1982] A. J. Dodd. The Core Model, volume 61 of London Mathematical Society Lecture Note Series. Cambridge University Press, 1982.

[Donder and Koepke, 1983] H.-D. Donder and P. G. Koepke. On the consistency strength of 'accessible' Jonsson cardinals and of the weak Chang conjecture. Annals of Pure and Applied Logic, 25:233-261, 1983.

[Donder et al., 1981] H.-D. Donder, R. B. Jensen, and B. J. Koppelberg. Some applications of the core model. In Set theory and model theory, volume 872 of Lecture Notes in Mathematics. Springer-Verlag, 1981.

[Ehrenfeucht and Mostowski, 1956] A. Ehrenfeucht and A. M. Mostowski. Models of axiomatic theories admitting automorphisms. Fundamenta Mathematicae, 43:50-68, 1956.

[Feng and Jech, 1998] Q. Feng and T. J. Jech. Projective stationary sets and a strong reflection principle. Journal of the London Mathematical Society, 58:271-283, 1998.

[Fleissner, 1982] W. G. Fleissner. If all normal moore spaces are metrizable, then there is an inner model with a measurable cardinal. Transactions of the American Mathematical Society, 273:365-373, 1982.

[Foreman and Kanamori, 2010] M. Foreman and A. Kanamori, editors. Handbook of Set Theory. Springer, 2010.

[Foreman and Woodin, 1991] M. Foreman and W. H. Woodin. The generalized continuum hypothesis can fail everywhere. Annals of Mathematics, 133:1-35, 1991.

[Foreman et al., 1984] M. Foreman, M. Magidor, and S. Shelah. Martin's Maximum, saturated ideals, and nonregular ultrafilters I. Annals of Mathematics, 127:1-47, 1984.

[Foreman, 1982] M. Foreman. Large cardinals and strong model theoretic-transfer properties. Transactions of the American Mathematical Society, 272:427-463, 1982.

[Foreman, 1983] M. Foreman. More saturated ideals. In A. S. Kechris, D. A. Martin, and Y. N. Moschovakis, editors, Cabal Seminar 79-81. Proceedings of the Caltech-UCLA Logic Seminar 1979-81, volume 1019 of Lecture Notes in Mathematics, pages 1-27. Springer-Verlag, 1983.

[Foreman, 1986] M. Foreman. Potent axioms. Transactions of the American Mathematical Society, 294:1-28, 1986.

[Gaifman, 1964] H. Gaifman. Measurable cardinals and constructible sets (abstract). Notices of the American Mathematical Society, 11:771, 1964.

[Gale and Stewart, 1953] D. Gale and F. M. Stewart. Infinite games with perfect information. In Contributions to the Theory of Games, vol. 2, Annals of Mathematical Studies \#28, pages 245-266. Princeton University Press, 1953. 
[Galvin and Hajnal, 1975] F. Galvin and A. Hajnal. Inequalities for cardinal powers. Annals of Mathematics, 101:491-498, 1975.

[Gitik and Shelah, 1989] M. Gitik and S. Shelah. Forcings with ideals and simple forcing notions. Israel Journal of Mathematics, 68:129-160, 1989.

[Gitik and Shelah, 1997] M. Gitik and S. Shelah. Less saturated ideals. Proceedings of the American Mathematical Society, 125:1523-1530, 1997.

[Gitik, 1980] M. Gitik. All cardinals can be singular. Israel Journal of Mathematics, 35:61-88, 1980.

[Gitik, 1984] M. Gitik. The nonstationary ideal on $\aleph_{2}$. Israel Journal of Mathematics, 48:257288, 1984.

[Gitik, 1995] M. Gitik. Some results on the nonstationary ideal. Israel Journal of Mathematics, 92:61-112, 1995.

[Gitik, 1997] M. Gitik. Some results on the nonstationary ideal. II. Israel Journal of Mathematics, 99:175-188, 1997.

[Harrington and Shelah, 1985] L. A. Harrington and S. Shelah. Some exact equiconsistency results in set theory. Notre Dame Journal of Formal Logic, 26:178-188, 1985.

[Jackson, 1988] S. Jackson. AD and the projective ordinals. In A. S. Kechris, D. A. Martin, and J. R. Steel, editors, Cabal Seminar 81-85. Proceedings, Caltech-UCLA Logic Seminar 1981-1985, volume 1333 of Lecture Notes in Mathematics, pages 117-220. Springer-Verlag, 1988.

[Jech and Prikry, 1976] T. J. Jech and K. L. Prikry. On ideals of sets and the power set operation. Bulletin of the American Mathematical Society, 82:593-595, 1976.

[Jech et al., 1980] T. J. Jech, M. Magidor, W. J. Mitchell, and K. L. Prikry. Precipitous ideals. The Journal of Symbolic Logic, 45:1-8, 1980.

[Jech, 1967] T. J. Jech. Non-provability of Souslin's hypothesis. Commentationes Mathematicae Universitatis Carolinae, 8:291-305, 1967.

[Jensen, 1968] R. B. Jensen. Souslin's hypothesis is incompatible with $V=L$ (abstract). Notices of the American Mathematical Society, 1968.

[Jensen, 1972] R. B. Jensen. The fine structure of the constructible hierarchy. Annals of Mathematical Logic, pages 229-308, 1972.

[Kanamori, 2003] A. Kanamori. The Higher Infinite. Large Cardinals in Set Theory from their Beginnings. Springer, 2003. Second edition.

[Kechris and Moschovakis, 1978] A. S. Kechris and Y. N. Moschovakis, editors. Cabal Seminar 76-77. Proceedings of the Caltech-UCLA Logic Seminar 1976-77, volume 689 of Lecture Notes in Mathematics. Springer-Verlag, 1978.

[Kechris et al., 1981] A. S. Kechris, D. A. Martin, and Y. N. Moschovakis, editors. Cabal Seminar 77-79. Proceedings of the Caltech-UCLA Logic Seminar 1977-79, volume 839 of Lecture Notes in Mathematics. Springer-Verlag, 1981.

[Kechris et al., 1983] A. S. Kechris, D. A. Martin, and Y. N. Moschovakis, editors. Cabal Seminar 79-81. Proceedings of the Caltech-UCLA Logic Seminar 1979-81, volume 1019 of Lecture Notes in Mathematics. Springer-Verlag, 1983.

[Kechris et al., 1988] A. S. Kechris, D. A. Martin, and J. R. Steel, editors. Cabal Seminar 8185. Proceedings of the Caltech-UCLA Logic Seminar 1981-85, volume 1333 of Lecture Notes in Mathematics. Springer-Verlag, 1988.

[Kunen and Vaughn, 1984] K. Kunen and J. E. Vaughn. Handbook of Set-Theoretic Topology. North-Holland, 1984

[Kunen, 1970] K. Kunen. Some applications of iterated ultrapowers in set theory. Annals of Mathematical Logic, 1:179-227, 1970.

[Kunen, 1971a] K. Kunen. Elementary embeddings and infinitary embeddings. The Journal of Symbolic Logic, 36:407-413, 1971.

[Kunen, 1971b] K. Kunen. On the GCH at measurable cardinals. In R. O. Gandy, editor, Logic Colloquium '69, pages 107-110. North-Holland, 1971.

[Kunen, 1978] K. Kunen. Saturated ideals. The Journal of Symbolic Logic, 43:65-76, 1978.

[Laver, 1976] R. Laver. On the consistency of Borel's Conjecture. Acta Mathematica, 137:151$169,1976$.

[Laver, 1978] R. Laver. Making the supercompactness of $\kappa$ indestructible under $\kappa$-directed closed forcing. Israel Journal of Mathematics, 29:385-388, 1978.

[Levinski et al., 1990] J.-P. Levinski, M. Magidor, and S. Shelah. Chang's Conjecture for $\aleph_{\omega}$ Israel Journal of Mathematics, 69:161-172, 1990. 
[Levy, 1965] A. Levy. A hierarchy of formulas in set theory. Memoirs of the American Mathematical Society, 57, 1965.

[Magidor, 1976] M. Magidor. How large is the first strongly compact cardinal? or: A study on identity crises. Annals of Mathematical Logic, 10:33-57, 1976.

[Magidor, 1977a] M. Magidor. On the singular cardinals problem. I. Israel Journal of Mathematics, 28:1-31, 1977 .

[Magidor, 1977b] M. Magidor. On the singular cardinals problem. II. Annals of Mathematics, 106:514-547, 1977.

[Magidor, 1978] M. Magidor. Changing cofinality of cardinals. Fundamenta Mathematicae, 99:61-71, 1978.

[Magidor, 1982] M. Magidor. Reflecting stationary sets. The Journal of Symbolic Logic, 47:755$771,1982$.

[Martin and Solovay, 1970] D. A. Martin and R. M. Solovay. Internal Cohen extensions. Annals of Mathematical Logic, 2:1443-178, 1970.

[Martin and Steel, 1988] D. A. Martin and J. R. Steel. Projective determinacy. Proceedings of the National Academy of Sciences USA, 85:6582-6586, 1988.

[Martin and Steel, 1989] D. A. Martin and J. R. Steel. A proof of Projective Determinacy. Journal of the American Mathematical Society, 2:1-73, 1989.

[Martin, 1970] D. A. Martin. Measurable cardinals and analytic games. Fundamenta Mathematicae, 66:287-291, 1970.

[Martin, 1975] D. A. Martin. Borel determinacy. Annals of Mathematics, 102:363-371, 1975.

[Martin, 1980] D. A. Martin. Infinite games. In O. Lehto, editor, Proceedings of the International Congress of Mathematicians, Helsinki 1978, pages 269-273. Academia Scientiarum Fennica, 1980

[Mitchell and Steel, 1994] W. J. Mitchell and J. R. Steel. Fine Structure and Iteration Trees, volume 3 of Lecture Notes in Logic. Springer, 1994.

[Mitchell et al., 1997] W. J. Mitchell, E. Schimmerling, and J. R. Steel. The covering lemma up to a Woodin cardinal. Annals of Pure and Applied Logic, 84:219-255, 1997.

[Mitchell, 1972] W. J. Mitchell. Aronszajn trees and the independence of the transfer property. Annals of Mathematical Logic, 5:21-46, 1972.

[Mitchell, 1974] W. J. Mitchell. Sets constructible from sequences of ultrafilters. The Journal of Symbolic Logic, 39:57-66, 1974.

[Mitchell, 1978] W. J. Mitchell. Hypermeasurable cardinals. In M. Boffa, D. van Dalen, and K. McAloon, editors, Logic Colloquium 'ry, Studies in Logic and the Foundations of Mathematics, pages 303-316. North-Holland, 1978.

[Mitchell, 1984] W. J. Mitchell. The core model for sequences of measures. I. Mathematical Proceedings of the Cambridge Philosophical Society, 95:229-260, 1984.

[Mitchell, 1985] W. J. Mitchell. The core model for sequences of measures. II. Available at http://www.math.ufl.edu/ wjm/papers, 1985.

[Mitchell, 1987] W. J. Mitchell. Applications of the covering lemma for sequences of measures. Transactions of the American Mathematical Society, 299:41-58, 1987.

[Moschovakis, 1971] Y. N. Moschovakis. Uniformization in a playful universe. Bulletin of the American Mathematical Society, 77:731-736, 1971.

[Mycielski and Steinhaus, 1962] J. Mycielski and H. Steinhaus. A mathematical axiom contradicting the axiom of choice. Bulletin de l'Académe Polonaise des Sciences, Série des Sciences Mathématiques, Astronomiques et Physiques, 10:1-3, 1962.

[Nyikos, 1980] P. J. Nyikos. A provisional solution to the normalMoore space problem. Proceedings of the American Mathematical Society, 78:429-435, 1980.

[Prikry, 1970] K. L. Prikry. Changing measurable cardinals into accessible cardinals. Dissertationes Mathematicae (Rozprawy Matematyczne), 68:5-52, 1970.

[Radin, 1982] L. B. Radin. Adding closed cofinal sequences to large cardinals. Annals of Mathematical Logic, 22:243-261, 1982.

[Schimmerling, 1994] E. Schimmerling. Combinatorial principles in the core model for one Woodin cardinal. Annals of Pure and Applied Logic, 74:153-201, 1994.

[Shelah and Woodin, 1990] S. Shelah and W. H. Woodin. Large cardinals imply that every reasonably definable set of reals is Lesbegue measurable. Israel Journal of Mathematics, 70:381-394, 1990

[Shelah, 1974] S. Shelah. Infinite abelian groups, Whitehead problem and some constructions. Israel Journal of Mathematics, 18:243-256, 1974. 
[Shelah, 1982] S. Shelah. Proper Forcing, volume 940 of Lecture Notes in Mathematics. Springer-Verlag, 1982.

[Shelah, 1983] S. Shelah. The singular cardinals problem: Independence results. In A. R. D. Mathias, editor, Surveys in Set Theory, volume 87 of London Mathematical Society Lecture Note Series, pages 116-134. Cambridge University Press, 1983.

[Shelah, 1984] S. Shelah. Can you take Solovay's inaccessible away? Israel Journal of Mathematics, 48:1-47, 1984.

[Shelah, 1986] S. Shelah. Around Classification Theory of Models, volume 1182 of Lecture Notes in Mathematics. Springer-Verlag, 1986.

[Shelah, 1987] S. Shelah. Semiproper Forcing Axiom implies Martin's Maximum but not PFA+. The Journal of Symbolic Logic, 52:360-367, 1987.

[Shelah, 1987a] S. Shelah. Iterated forcing and normal ideals on $\omega_{1}$. Israel Journal of Mathematics, 60:345-380, 1987.

[Shelah, 1994] S. Shelah. Cardinal Arithmetic, volume 29 of Oxford Logic Guides. Oxford University Press, 1994.

[Shelah, 1998] S. Shelah. Proper and Improper Forcing, volume 29 of Perspectives in Mathematical Logic. Springer-Verlag, 1998.

[Shoenfield, 1961] J. R. Shoenfield. The problem of predicativity. In Y. Bar-Hillel, E. I. J. Poznanski, M. O. Rabin, and A. Robinson, editors, Essays on the Foundations of Mathematics, pages 132-139. Magnes Press, 1961.

[Shoenfield, 1971] J. R. Shoenfield. Unramified forcing. In D. S. Scott, editor, Axiomatic Set Theory, volume 13(1) of Proceedings of Symposia in Pure Mathematics, pages 357-381. American Mathematical Society, Providence, 1971.

[Silver, 1971a] J. H. Silver. The consistency of the GCH with the existence of a measurable cardinal. In Dana S. Scott, editor, Axiomatic Set Theory, Proceedings of Symposia in Pure Mathematics, pages 391-395. American Mathematical Society, Providence, 1971.

[Silver, 1971b] J. H. Silver. The independence of Kurepa's conjecture and two-cardinal conjectures in model theory. In D. S. Scott, editor, Axiomatic Set Theory, volume 13(1) of Proceedings of Symposia in Pure Mathematics, pages 383-390. American Mathematical Society, Providence, 1971.

[Silver, 1971c] J. H. Silver. Some applications of model theory in set theory. Annals of Mathematical Logic, 3:45-110, 1971.

[Silver, 1975] J. H. Silver. On the singular cardinals problem. In Proceedings of the International Congress of Mathematicians, Vancouver, B. C., 1974, volume 1, pages 265-268. Canadian Mathematical Congress, 1975.

[Solovay and Tennenbaum, 1971] R. M. Solovay and S. Tennenbaum. Internal Cohen extensions and Souslin's problem. Annals of Mathematics, 94:201-245, 1971.

[Solovay et al., 1978] R. M. Solovay, W. N. Reinhardt, and A. Kanamori. Strong axioms of infinity and elementary embeddings. Annals of Mathematical Logic, 13:73-116, 1978.

[Solovay, 1966] R. M. Solovay. Real-valued measurable cardinals (abstract). Notices of the American Mathematical Society, 13:721, 1966.

[Solovay, 1967] R. M. Solovay. A nonconstructible $\Delta_{3}^{1}$ set of integers. Transactions of the American Mathematical Society, 127:50-75, 1967.

[Solovay, 1971] R. M. Solovay. Real-valued measurable cardinals. In Dana S. Scott, editor, $A x-$ iomatic Set Theory, volume 13(1) of Proceedings of Symposia in Pure Mathematics. American Mathematical Society, Providence, 1971.

[Steel, 1996] J. R. Steel. The Core Model Iterability Problem. Springer, 1996.

[Stewart, 1966] D. H. Stewart. Master's thesis, University of Bristol, 1966.

[Tennenbaum, 1968] S. Tennenbaum. Souslin's problem. Proceedings of the National Academy of Sciences USA, 59:60-64, 1968.

[Todorcevic, 1984] S. Todorcevic. A note on the Proper Forcing Axiom. In James E. Baumgartner, D. Anthony Martin, and Saharon Shelah, editors, Axiomatic Set Theory, volume 31 of Contemporary Mathematics, pages 209-218. American Mathematical Society, 1984.

[Todorcevic, 2007] S. Todorcevic. Walks on Ordinals and their Characteristics, volume 263 of Progress in Mathematics. Birkhäuser, 2007.

[Woodin, 1990] W. H. Woodin. Supercompact cardinals, sets of reals, and weakly homogeneous trees. Proceedings of the National Academy of Sciences USA, 85:6587-6591, 1990. 
[Woodin, 1999] W. H. Woodin. The Axiom of Determinacy, Forcing Axioms and the Nonstationary Ideal, volume 1 of de Gruyter Series in Logic and Its Applications. de Gruyter, 1999.

[Woodin, 2001a] W. H. Woodin. The Continuum Hypothesis. I. Notices of the American Mathematical Society, 48:567-576, 2001.

[Woodin, 2001b] W. H. Woodin. The Continuum Hypothesis. II. Notices of the American Mathematical Society, 48:681-690, 2001. 